DOI 10.4171/JEMS/222

Stefan Ivanov · Ivan Minchev · Dimiter Vassilev

\title{
Extremals for the Sobolev inequality on the seven-dimensional quaternionic Heisenberg group and the quaternionic contact Yamabe problem
}

Received January 10, 2008 and in revised form July 22, 2008

\begin{abstract}
A complete solution to the quaternionic contact Yamabe problem on the seven-dimensional sphere is given. Extremals for the Sobolev inequality on the seven-dimensional Heisenberg group are explicitly described and the best constant in the $L^{2}$ Folland-Stein embedding theorem is determined.
\end{abstract}

Keywords. Yamabe equation, quaternionic contact structures, Einstein structures

\section{Introduction}

It is well known that the sphere at infinity of any non-compact symmetric space $M$ of rank one carries a natural Carnot-Carathéodory structure (see $[\mathrm{M}, \mathrm{P}]$ ). A quaternionic contact (qc) structure [Biq1, Biq2] appears naturally as the conformal boundary at infinity of the quaternionic hyperbolic space. In this paper, following Biquard, a quaternionic contact structure ( $q c$ structure) on a real $(4 n+3)$-dimensional manifold $M$ is a codimension three distribution $H$ locally given as the kernel of an $\mathbb{R}^{3}$-valued 1-form $\eta=\left(\eta_{1}, \eta_{2}, \eta_{3}\right)$ such that the three 2-forms $\left.d \eta_{i}\right|_{H}$ are the fundamental forms of a quaternionic structure on $H$. This means that there exists a Riemannian metric $g$ on $H$ and three local almost complex structures $I_{i}$ on $H$ satisfying the commutation relations of the imaginary quaternions, $I_{1} I_{2} I_{3}=-1$, such that $\left.d \eta_{i}\right|_{H}=2 g\left(I_{i} \cdot, \cdot\right)$. The 1 -form $\eta$ is determined up to a conformal factor and the action of $S O(3)$ on $\mathbb{R}^{3}$, and therefore $H$ is equipped with a conformal class $[g]$ of Riemannian metrics and a 2-sphere bundle of almost complex structures, the quaternionic bundle $\mathbb{Q}$. The 2 -sphere bundle of one forms determines uniquely the associated metric and a conformal change of the metric is equivalent to a conformal change of the 1-forms. To every metric in the fixed conformal class one can associate a

S. Ivanov: Faculty of Mathematics and Informatics, University of Sofia, blvd. James Bourchier 5, 1164, Sofia, Bulgaria; e-mail: ivanovsp@fmi.uni-sofia.bg

I. Minchev: University of Sofia, Sofia, Bulgaria, and Institut für Mathematik, Humboldt Universität zu Berlin, Unter den Linden 6, D-10099 Berlin, Germany; e-mail: minchevim@yahoo.com, minchev@fmi.uni-sofia.bg

D. Vassilev: Department of Mathematics and Statistics, University of New Mexico, Albuquerque, NM 87131-0001, USA and University of California at Riverside, Riverside, CA 92521, USA; e-mail: vassilev@math.unm.edu

Mathematics Subject Classification (2010): 58G30, 53C17 
linear connection preserving the qc structure (see [Biq1]), which we shall call the Biquard connection.

If the first Pontryagin class of $M$ vanishes then the 2-sphere bundle of $\mathbb{R}^{3}$-valued 1forms is trivial [AK], i.e. there is a globally defined 3-contact form $\eta$ that annihilates $H$; we denote the corresponding QC manifold by $(M, \eta)$. In this case the 2 -sphere of associated almost complex structures is also globally defined on $H$.

Examples of QC manifolds are given in [Biq1, Biq2, IMV, D2]. In particular, any totally umbilic hypersurface of a quaternionic Kähler or hyperkähler manifold carries such a structure [IMV]. A basic example is provided by any 3-Sasakian manifold which can be defined as a $(4 n+3)$-dimensional Riemannian manifold whose Riemannian cone is a hyperkähler manifold. It was shown in [IMV] that the torsion endomorphism of the Biquard connection is the obstruction for a given qc-structure to be locally 3-Sasakian, up to multiplication with a constant factor and an $S O(3)$-matrix.

To a fixed metric in the conformal class of metrics on the horizontal space one associates the scalar curvature of the associated Biquard connection, called the qc-scalar curvature. Guided by the real (Riemannian) and complex (CR) cases, the quaternionic contact Yamabe problem is: given a compact QC manifold $(M, \eta)$, find a conformal 3contact form for which the qc-scalar curvature is constant.

In the present paper we provide a solution of this problem on the seven-dimensional sphere equipped with its natural quaternionic contact structure. The spheres are important examples of locally quternionic conformally flat qc structures characterized locally in [IV] by the vanishing of a curvature-type tensor invariant and from the point of view of the Yamabe problem play a role similar to their Riemannian and CR counterparts. The question reduces to the solvability of the Yamabe equation (3.4) below. Taking the conformal factor in the form $\bar{\eta}=u^{4 /(Q-2)} \eta, Q=4 n+6$, turns 3.4 into the equation

$$
\mathcal{L} u \equiv 4 \frac{Q+2}{Q-2} \Delta u-u \text { Scal }=-u^{2^{*}-1} \overline{\mathrm{Scal}},
$$

where $\Delta$ is the horizontal sub-Laplacian, $\Delta h=\operatorname{tr}^{g}(\nabla d h)$, Scal and $\overline{\text { Scal }}$ are the qc-scalar curvatures respectively of $(M, \eta)$ and $(M, \bar{\eta})$, and $2^{*}=2 Q /(Q-2)$, with $Q=4 n+6$ the homogeneous dimension. On a compact quaternionic contact manifold $M$ with a fixed conformal class $[\eta]$ the Yamabe equation characterizes the non-negative extremals of the Yamabe functional defined by

$$
\Upsilon(u)=\int_{M}\left(4 \frac{Q+2}{Q-2}|\nabla u|^{2}+\operatorname{Scal} u^{2}\right) d v_{g}, \quad \int_{M} u^{2^{*}} d v_{g}=1, \quad u>0 .
$$

Considering $M$ equipped with a fixed qc structure, hence, a conformal class [ $\eta]$, the Yamabe constant is defined as the infimum

$$
\lambda(M) \equiv \lambda(M,[\eta])=\inf \left\{\Upsilon(u): \int_{M} u^{2^{*}} d v_{g}=1, u>0\right\} .
$$

Here $d v_{g}$ denotes the Riemannian volume form of the Riemannian metric on $M$ extending in a natural way the horizontal metric associated to $\eta$. 
When the Yamabe constant $\lambda(M)$ is less than that of the quaternionic sphere with its standard qc structure the existence of solutions to the quaternionic contact Yamabe problem is shown in [W] (see also [JL1]). We consider the Yamabe problem on the standard unit $(4 n+3)$-dimensional quaternionic sphere. The standard 3-Sasaki structure on the sphere is a qc-Einstein structure $\tilde{\eta}$ having constant qc-scalar curvature $\widetilde{\mathrm{Scal}}=16 n(n+2)$. Its images under conformal quaternionic contact automorphisms have again constant qcscalar curvature. In [IMV] we conjectured that these are the only solutions to the Yamabe problem on the quaternionic sphere. The purpose of this paper is to prove this conjecture when the dimension is equal to seven, i.e., $n=1$.

Theorem 1.1. Let $\tilde{\eta}=\frac{1}{2 h} \eta$ be a conformal deformation of the standard qc structure $\tilde{\eta}$ on the quaternionic unit sphere $S^{7}$. If $\eta$ has constant qc-scalar curvature, then up to a multiplicative constant, $\eta$ is obtained from $\tilde{\eta}$ by a conformal quaternionic contact automorphism. In particular, $\lambda\left(S^{7}\right)=48(4 \pi)^{1 / 5}$ and this minimum value is achieved only by $\tilde{\eta}$ and its images under conformal quaternionic contact automorphisms.

In [IMV] a weaker result was shown, namely the conclusion holds (in all dimensions) provided the vertical space of $\eta$ is integrable. We recall the definition of conformal quaternionic contact automorphisms in Definition 2.1

Another motivation for studying the Yamabe equation comes from its connection with the determination of the norm and extremals in a relevant Sobolev-type embedding on the quaternionic Heisenberg group $\boldsymbol{G}(\mathbb{H})$ ([GV1], [Va1], [Va2]). As is well known, the Yamabe equation is essentially the Euler-Lagrange equation of the extremals for the $L^{2}$ case of such embedding results. In the present setting we have the following theorem due to Folland and Stein $[\mathrm{FSt}]$.

Theorem 1.2 (Folland and Stein). Let $\Omega \subset G$ be an open set in a Carnot group $G$ of homogeneous dimension $Q$ and Haar measure $d H$. For any $1<p<Q$ there exists $S_{p}=S_{p}(G)>0$ such that for $u \in C_{0}^{\infty}(\Omega)$,

$$
\left(\int_{\Omega}|u|^{p^{*}} d H(g)\right)^{1 / p^{*}} \leq S_{p}\left(\int_{\Omega}|X u|^{p} d H(g)\right)^{1 / p},
$$

where $|X u|=\sum_{j=1}^{m}\left|X_{j} u\right|^{2}$ with $X_{1}, \ldots, X_{m}$ denoting a basis of the first layer of $G$ and $p^{*}=p Q /(Q-p)$.

Let $S_{p}$ be the best constant in the Folland-Stein inequality, i.e., the smallest constant for which (1.1) holds. The second result of this paper is the following theorem, which determines the extremals and the best constant in Theorem 1.2 when $p=2$ for the case of the seven-dimensional quaternionic Heisenberg group $G(\mathbb{H})$. As a manifold, $G(\mathbb{H})=$ $\mathbb{H} \times \operatorname{Im} \mathbb{H}$ with the group law given by

$$
\left(q^{\prime}, \omega^{\prime}\right)=\left(q_{0}, \omega_{0}\right) \circ(q, \omega)=\left(q_{0}+q, \omega+\omega_{0}+2 \operatorname{Im} q_{0} \bar{q}\right),
$$

where $q, q_{0} \in \mathbb{H}$ and $\omega, \omega_{0} \in \operatorname{Im} \mathbb{H}$. The standard quaternionic contact (qc) structure is defined by the left-invariant quaternionic contact form $\tilde{\Theta}=\left(\tilde{\Theta}_{1}, \tilde{\Theta}_{2}, \tilde{\Theta}_{3}\right)=$ $\frac{1}{2}\left(d \omega-q^{\prime} \cdot d \bar{q}^{\prime}+d q^{\prime} \cdot \bar{q}^{\prime}\right)$, where $\cdot$ denotes quaternion multiplication. 
Theorem 1.3. Let $\boldsymbol{G}(\mathbb{H})=\mathbb{H} \times \operatorname{Im} \mathbb{H}$ be the seven-dimensional quaternionic Heisenberg group. The best constant in the $L^{2}$ Folland-Stein embedding theorem is

$$
S_{2}=\frac{2 \sqrt{3}}{\pi^{3 / 5}},
$$

An extremal is given by the function

$$
v=\frac{2^{11} \sqrt{3}}{\pi^{3 / 5}}\left[\left(1+|q|^{2}\right)^{2}+|\omega|^{2}\right]^{-2}, \quad(q, \omega) \in G(\mathbb{H}) .
$$

Any other non-negative extremal is obtained from $v$ by translations (5.10) and dilations 5.11.

Our result confirms the Conjecture made after [GV1, Theorem 1.1]. In [GV1, Theorem 1.6] the above theorem is proved in all dimensions, but with the assumption of partial symmetry. Here with a completely different method we show that the symmetry assumption is superfluous in the case of the first quaternionic Heisenberg group. On the other hand, in [IMV] we proved Theorem 1.1 in all dimensions, but with the extra assumption of the integrability of the vertical distribution. In the present paper we remove the extra integrability assumption in dimension seven. A key step is the establishment of a suitable divergence formula, Theorem 4.4 (see [JL2] for the CR case and [Ob], [LP] for the Riemannian case). With the help of this divergence formula we show that the 'new' structure is also qc-Einstein, thus we reduce the Yamabe problem on the 7-sphere from solving the non-linear Yamabe equation to a geometrical system of differential equations describing the qc-Einstein structures conformal to the standard one. Invoking the (quaternionic) Cayley transform, which is a contact conformal diffeomorphism, [IMV], we turn the question to the corresponding system on the quaternionic Heisenberg group. On the latter all global solutions are explicitly described in [IMV] and this allows us to conclude the proof of our results.

Remark 1.4. With the left-invariant basis of Theorem 1.3 the Heisenberg group $G(\mathbb{H})$ is not a group of Heisenberg type. If we consider $\boldsymbol{G}(\mathbb{H})$ as a group of Heisenberg type then the best constant in the $L^{2}$ Folland-Stein embedding theorem is (cf. [GV1, Theorem 1.6])

$$
S_{2}=\frac{15^{1 / 10}}{\pi^{2 / 5} 2 \sqrt{2}},
$$

and an extremal is given by the function

$$
F(q, \omega)=\gamma\left[\left(1+|q|^{2}\right)^{2}+16|\omega|^{2}\right]^{-2}, \quad(q, \omega) \in \boldsymbol{G}(\mathbb{H}),
$$

where

$$
\gamma=32 \pi^{-17 / 50} 2^{1 / 5} 15^{2 / 5} .
$$

Organization of the paper. The paper uses some results from [IMV]. In order to make the present paper self-contained, in Section 2 we give a review of the notion of a quaternionic contact structure and collect formulas and results from [IMV] that will be used in the subsequent sections. 
Sections 3 and 4 are of technical nature. In the former we find some transformation formulas for relevant tensors, while in the latter we prove certain divergence formulas. The key result is Theorem 4.4 , with the help of which in the last section we prove the main theorems.

Convention 1.5. We use the following conventions:

- $\left\{e_{1}, \ldots, e_{4 n}\right\}$ denotes an orthonormal basis of the horizontal space $H$.

- The summation convention over repeated vectors from the basis $\left\{e_{1}, \ldots, e_{4 n}\right\}$ will be used. For example, for a $(0,4)$-tensor $P$, the formula $k=P\left(e_{b}, e_{a}, e_{a}, e_{b}\right)$ means

$$
k=\sum_{a, b=1}^{4 n} P\left(e_{b}, e_{a}, e_{a}, e_{b}\right) .
$$

- The triple $(i, j, k)$ denotes any cyclic permutation of $(1,2,3)$.

\section{Quaternionic contact manifolds}

In this section we will briefly review the basic notions of quaternionic contact geometry and recall some results from [Biq1] and [IMV].

For the purposes of this paper, a quaternionic contact $(\mathrm{QC})$ manifold $(M, g, \mathbb{Q})$ is a $(4 n+3)$-dimensional manifold $M$ with a codimension three distribution $H$ equipped with a metric $g$ and an $\operatorname{Sp}(n) \operatorname{Sp}(1)$ structure, i.e., we have

(i) a 2-sphere bundle $\mathbb{Q}$ over $M$ of almost complex structures such that $\mathbb{Q}=$ $\left\{a I_{1}+b I_{2}+c I_{3}: a^{2}+b^{2}+c^{2}=1\right\}$, where the almost complex structures $I_{s}:$ $H \rightarrow H, I_{s}^{2}=-1, s=1,2,3$, satisfy the commutation relations of the imaginary quaternions $I_{1} I_{2}=-I_{2} I_{1}=I_{3}$;

(ii) $H$ is the kernel of a 1 -form $\eta=\left(\eta_{1}, \eta_{2}, \eta_{3}\right)$ with values in $\mathbb{R}^{3}$ and the following compatibility condition holds:

$$
2 g\left(I_{s} X, Y\right)=d \eta_{s}(X, Y), \quad s=1,2,3, X, Y \in H .
$$

Given a quaternionic contact manifold we shall denote by $\eta$ any associated contact form. The associated contact form is determined up to an $S O$ (3)-action, namely if $\Psi \in$ $S O$ (3) with smooth functions as entries then $\Psi \eta$ is again a contact form satisfying the above compatibility condition (rotating also the almost complex structures). On the other hand, if we consider the conformal class $[g]$, the associated contact forms are determined up to multiplication with a positive function $\mu$ and an $S O$ (3)-action, namely if $\Psi \in$ $S O$ (3) then $\mu \Psi \eta$ is a contact form associated with a metric in the conformal class $[g]$.

We shall denote by $(M, \eta)$ a QC manifold with a fixed globally defined contact form. A special phenomenon here, noted in [Biq1], is that the 3-contact form $\eta$ determines the quaternionic structure and the metric on the horizontal bundle in a unique way.

A QC manifold $(M, \bar{g}, \mathbb{Q})$ is called conformal to $(M, g, \mathbb{Q})$ if $\bar{g} \in[g]$. In that case, if $\bar{\eta}$ is a corresponding associated 1 -form with complex structures $\bar{I}_{s}, s=1,2,3$, we have $\bar{\eta}=\mu \Psi \eta$ for some $\Psi \in S O$ (3) with smooth functions as entries and a positive function $\mu$. In particular, starting with a QC manifold $(M, \eta)$ and defining $\bar{\eta}=\mu \eta$ we obtain a QC manifold $(M, \bar{\eta})$ conformal to the original one. 
Definition 2.1. A diffeomorphism $\phi$ of a QC manifold $(M,[g], \mathbb{Q})$ is called a conformal quaternionic contact automorphism (conformal qc-automorphism) if $\phi$ preserves the QC structure, i.e.

$$
\phi^{*} \eta=\mu \Psi \cdot \eta
$$

for some positive smooth function $\mu$ and some matrix $\Psi \in S O$ (3) with smooth functions as entries and $\eta=\left(\eta_{1}, \eta_{2}, \eta_{3}\right)^{t}$ is a local 1-form considered as a column vector of three 1 -forms as entries.

Any endomorphism $\Psi$ of $H$ can be decomposed with respect to the quaternionic structure $(\mathbb{Q}, g)$ uniquely into $\operatorname{Sp}(n)$-invariant parts as follows: $\Psi=\Psi^{+++}+\Psi^{+--}+$ $\Psi^{-+-}+\Psi^{--+}$, where $\Psi^{+++}$commutes with all three $I_{i}, \Psi^{+--}$commutes with $I_{1}$ and anti-commutes with the other two etc. The two $\operatorname{Sp}(n) \operatorname{Sp}(1)$-invariant components are given by

$$
\Psi_{[3]}=\Psi^{+++}, \quad \Psi_{[-1]}=\Psi^{+--}+\Psi^{-+-}+\Psi^{--+} .
$$

Denoting the corresponding $(0,2)$-tensor via $g$ by the same letter one sees that the $\mathrm{Sp}(n) \mathrm{Sp}(1)$-invariant components are the projections on the eigenspaces of the Casimir operator

$$
\dagger=I_{1} \otimes I_{1}+I_{2} \otimes I_{2}+I_{3} \otimes I_{3}
$$

corresponding, respectively, to the eigenvalues 3 and -1 (see [CSal]). If $n=1$ then the space of symmetric endomorphisms commuting with all $I_{i}, i=1,2,3$, is 1-dimensional, i.e. the [3]-component of any symmetric endomorphism $\Psi$ on $H$ is proportional to the identity, $\Psi_{[3]}=(\operatorname{tr}(\Psi) / 4) \operatorname{Id}_{\mid H}$.

On a quaternionic contact manifold there exists a canonical connection defined in [Biq1] when $4 n+3>7$, and in [D1] in the 7-dimensional case.

Theorem 2.2 ([Biq1] $)$. Let $(M, g, \mathbb{Q})$ be a quaternionic contact manifold of dimension $4 n+3>7$ and a fixed metric $g$ on $H$ in the conformal class $[g]$. Then there exists $a$ unique connection $\nabla$ with torsion $T$ on $M^{4 n+3}$ and a unique supplementary subspace $V$ to $H$ in $T M$ such that:

(i) $\nabla$ preserves the decomposition $H \oplus V$ and the metric $g$;

(ii) for $X, Y \in H$, one has $T(X, Y)=-[X, Y]_{\mid V}$;

(iii) $\nabla$ preserves the $\operatorname{Sp}(n) \operatorname{Sp}(1)$-structure on $H$, i.e., $\nabla g=0$ and $\nabla \mathbb{Q} \subset \mathbb{Q}$;

(iv) for $\xi \in V$, the endomorphism $T(\xi, \cdot)_{\mid H}$ of $H$ lies in $(\operatorname{sp}(n) \oplus \operatorname{sp}(1))^{\perp} \subset \operatorname{gl}(4 n)$;

(v) the connection on $V$ is induced by the natural identification $\varphi$ of $V$ with the subspace $\mathrm{sp}(1)$ of the endomorphisms of $H$, i.e. $\nabla \varphi=0$.

We shall call the above connection the Biquard connection. Biquard [Biq1] also described the supplementary subspace $V$ explicitly, namely, locally $V$ is generated by vector fields $\left\{\xi_{1}, \xi_{2}, \xi_{3}\right\}$ such that

$$
\left.\left.\left.\eta_{s}\left(\xi_{k}\right)=\delta_{s k}, \quad\left(\xi_{s}\right\lrcorner d \eta_{s}\right)_{\mid H}=0, \quad\left(\xi_{s}\right\lrcorner d \eta_{k}\right)_{\mid H}=-\left(\xi_{k}\right\lrcorner d \eta_{s}\right)_{\mid H} .
$$

The vector fields $\xi_{1}, \xi_{2}, \xi_{3}$ are called Reeb vector fields or fundamental vector fields. 
If the dimension of $M$ is seven, the conditions (2.3) do not always hold. Duchemin shows in [D1] that if we assume, in addition, the existence of Reeb vector fields as in 2.3, then Theorem 2.2 holds. Henceforth, by a qc structure in dimension 7 we shall mean a qc structure satisfying 2.3 .

Notice that equations (2.3) are invariant under the natural $S O(3)$ action. Using the triple of Reeb vector fields we extend $g$ to a metric on $M$ by requiring $\operatorname{span}\left\{\xi_{1}, \xi_{2}, \xi_{3}\right\}=$ $V \perp H$ and $g\left(\xi_{s}, \xi_{k}\right)=\delta_{s k}$. The extended metric does not depend on the action of $S O$ (3) on $V$, but it changes in an obvious manner if $\eta$ is multiplied by a conformal factor. Clearly, the Biquard connection preserves the extended metric on $T M, \nabla g=0$. We shall also extend the quaternionic structure by setting $I_{S \mid V}=0$. The fundamental 2 -forms $\omega_{i}$, $i=1,2,3$, of the quaternionic structure $Q$ are defined by

$$
\left.2 \omega_{i \mid H}=d \eta_{i \mid H}, \quad \xi\right\lrcorner \omega_{i}=0, \quad \xi \in V .
$$

Due to 2.4, the torsion restricted to $H$ has the form

$$
T(X, Y)=-[X, Y]_{\mid V}=2 \sum_{s=1}^{3} \omega_{s}(X, Y) \xi_{s}, \quad X, Y \in H
$$

The properties of the Biquard connection are encoded in the properties of the torsion endomorphism $T_{\xi}=T(\xi, \cdot): H \rightarrow H, \xi \in V$. Decomposing the endomorphism $T_{\xi} \in$ $(\operatorname{sp}(n)+\operatorname{sp}(1))^{\perp}$ into its symmetric part $T_{\xi}^{0}$ and skew-symmetric part $b_{\xi}, T_{\xi}=T_{\xi}^{0}+b_{\xi}$, we summarize the description of the torsion due to O. Biquard in the following proposition.

Proposition 2.3 ([|Biq1] $)$. The torsion $T_{\xi}$ is completely trace-free,

$$
\operatorname{tr} T_{\xi}=g\left(T_{\xi}\left(e_{a}\right), e_{a}\right)=0, \quad \operatorname{tr} T_{\xi} \circ I=g\left(T_{\xi}\left(e_{a}\right), I e_{a}\right)=0, \quad I \in Q,
$$

where $e_{1}, \ldots, e_{4 n}$ is an orthonormal basis of $H$. If we decompose the torsion into symmetric and antisymmetric parts, $T_{\xi_{i}}=T_{\xi_{i}}^{0}+b_{\xi_{i}}, i=1,2,3$, the symmetric part of the torsion has the properties

$$
\begin{gathered}
T_{\xi_{i}}^{0} I_{i}=-I_{i} T_{\xi_{i}}^{0}, \\
I_{2}\left(T_{\xi_{2}}^{0}\right)^{+--}=I_{1}\left(T_{\xi_{1}}^{0}\right)^{-+-}, \quad I_{3}\left(T_{\xi_{3}}^{0}\right)^{-+-}=I_{2}\left(T_{\xi_{2}}^{0}\right)^{--+}, \quad I_{1}\left(T_{\xi_{1}}^{0}\right)^{--+}=I_{3}\left(T_{\xi_{3}}^{0}\right)^{+--},
\end{gathered}
$$

and the skew-symmetric part can be represented as

$$
b_{\xi_{i}}=I_{i} u
$$

where $u$ is a traceless symmetric $(1,1)$-tensor on $H$ which commutes with $I_{1}, I_{2}, I_{3}$.

If $n=1$ then the tensor $u$ vanishes identically, $u=0$, and the torsion is a symmetric tensor, $T_{\xi}=T_{\xi}^{0}$.

The covariant derivative of the quaternionic contact structure with respect to the Biquard connection and the covariant derivative of the distribution $V$ are given by

$$
\nabla I_{i}=-\alpha_{j} \otimes I_{k}+\alpha_{k} \otimes I_{j}, \quad \nabla \xi_{i}=-\alpha_{j} \otimes \xi_{k}+\alpha_{k} \otimes \xi_{j}
$$


where the $\operatorname{sp}(1)$-connection 1-forms $\alpha_{s}$ on $H$ are given by [Biq1]

$$
\alpha_{i}(X)=d \eta_{k}\left(\xi_{j}, X\right)=-d \eta_{j}\left(\xi_{k}, X\right), \quad X \in H, \xi_{i} \in V,
$$

while the $\operatorname{sp}(1)$-connection 1 -forms $\alpha_{s}$ on the vertical space $V$ are calculated in [IMV] to be

$$
\begin{aligned}
\alpha_{i}\left(\xi_{s}\right)= & d \eta_{s}\left(\xi_{j}, \xi_{k}\right) \\
& -\delta_{i s}\left(\frac{\mathrm{Scal}}{16 n(n+2)}+\frac{1}{2}\left(d \eta_{1}\left(\xi_{2}, \xi_{3}\right)+d \eta_{2}\left(\xi_{3}, \xi_{1}\right)+d \eta_{3}\left(\xi_{1}, \xi_{2}\right)\right)\right),
\end{aligned}
$$

where $s \in\{1,2,3\}$. The vanishing of the $\operatorname{sp}(1)$-connection 1-forms on $H$ is equivalent to the vanishing of the torsion endomorphism of the Biquard connection (see [IMV]).

\subsection{The qc-Einstein condition and Bianchi identities}

We explain briefly the consequences of the Bianchi identities and the notion of qc-Einstein manifold introduced in [IMV] since it plays a crucial role in solving the Yamabe equation in the quaternionic seven-dimensional sphere. For more details see [IMV].

Let $\left.R=[\nabla, \nabla]-\nabla_{[,},\right]$be the curvature tensor of $\nabla$. The Ricci tensor and the scalar curvature Scal of the Biquard connection, called qc-Ricci tensor and qc-scalar curvature, respectively, are defined by

$\operatorname{Ric}(X, Y)=g\left(R\left(e_{a}, X\right) Y, e_{a}\right), \quad X, Y \in H, \quad \operatorname{Scal}=\operatorname{Ric}\left(e_{a}, e_{a}\right)=g\left(R\left(e_{b}, e_{a}\right) e_{a}, e_{b}\right)$.

According to [Biq1] the Ricci tensor restricted to $H$ is a symmetric tensor. If the tracefree part of the qc-Ricci tensor is zero we call the quaternionic structure a qc-Einstein manifold [IMV]. It is shown in [IMV] that the qc-Ricci tensor is completely determined by the components of the torsion. First, recall the notion of the $\operatorname{Sp}(n) \operatorname{Sp}(1)$-invariant trace-free symmetric 2-tensors $T^{0}, U$ on $H$ introduced in [IMV] by

$$
T^{0}(X, Y):=g\left(\left(T_{\xi_{1}}^{0} I_{1}+T_{\xi_{2}}^{0} I_{2}+T_{\xi_{3}}^{0} I_{3}\right) X, Y\right), \quad U(X, Y):=g(u X, Y), \quad X, Y \in H .
$$

The tensor $T^{0}$ belongs to the [-1]-eigenspace while $U$ is in the [3]-eigenspace of the operator $\dagger$ given by $(2.2)$, i.e.,

$$
\begin{array}{r}
T^{0}(X, Y)+T^{0}\left(I_{1} X, I_{1} Y\right)+T^{0}\left(I_{2} X, I_{2} Y\right)+T^{0}\left(I_{3} X, I_{3} Y\right)=0, \\
3 U(X, Y)-U\left(I_{1} X, I_{1} Y\right)-U\left(I_{2} X, I_{2} Y\right)-U\left(I_{3} X, I_{3} Y\right)=0 .
\end{array}
$$

Theorem 1.3, Theorem 3.12 and Corollary 3.14 in [IMV] imply:

Theorem 2.4 ([IMV $])$. Let $\left(M^{4 n+3}, g, \mathbb{Q}\right)$ be a quaternionic contact $(4 n+3)$-dimensional manifold, $n>1$. For any $X, Y \in H$ the qc-Ricci tensor and the qc-scalar curvature satisfy

$$
\begin{aligned}
\operatorname{Ric}(X, Y) & =(2 n+2) T^{0}(X, Y)+(4 n+10) U(X, Y)+\frac{\text { Scal }}{4 n} g(X, Y), \\
\text { Scal } & =-8 n(n+2) g\left(T\left(\xi_{1}, \xi_{2}\right), \xi_{3}\right)
\end{aligned}
$$

For $n=1$ the above formulas hold with $U=0$. 
In particular, the qc-Einstein condition is equivalent to the vanishing of the torsion endomorphism of the Biquard connection. If Scal $\neq 0$ the latter holds exactly when the qc-structure is 3-Sasakian up to multiplication by a constant and an SO(3)-matrix with smooth entries.

For the last part of the above theorem, we recall that a $(4 n+3)$-dimensional Riemannian manifold $(M, g)$ is called 3-Sasakian if the cone metric $g_{N}=t^{2} g+d t^{2}$ on $N=M \times \mathbb{R}^{+}$ is a hyperkähler metric, namely, it has holonomy contained in $\operatorname{Sp}(n+1)$.

The Ricci 2-forms $\rho_{s}, s=1,2,3$, of a quaternionic contact structure are defined by

$$
4 n \rho_{s}(B, C)=g\left(R(B, C) e_{a}, I_{s} e_{a}\right), \quad B, C \in \Gamma(T M) .
$$

For ease of reference, in the following theorem we summarize the properties of the Ricci 2-forms, the scalar curvature and the torsion evaluated on the vertical space established in Lemma 3.11, Corollary 3.14, Proposition 4.3 and Proposition 4.4 of [IMV] .

Theorem 2.5 ([IMV]). The Ricci 2-forms satisfy

$$
\begin{gathered}
\rho_{1}(X, Y)=2 g\left(\left(T_{\xi_{2}}^{0}\right)^{--+} I_{3} X, Y\right)-2 g\left(I_{1} u X, Y\right)-\frac{\text { Scal }}{8 n(n+2)} \omega_{1}(X, Y), \\
\rho_{2}(X, Y)=2 g\left(\left(T_{\xi_{3}}^{0}\right)^{+--} I_{1} X, Y\right)-2 g\left(I_{2} u X, Y\right)-\frac{\text { Scal }}{8 n(n+2)} \omega_{2}(X, Y), \\
\rho_{3}(X, Y)=2 g\left(\left(T_{\xi_{1}}^{0}\right)^{-+-} I_{2} X, Y\right)-2 g\left(I_{3} u X, Y\right)-\frac{\text { Scal }}{8 n(n+2)} \omega_{3}(X, Y) . \\
\rho_{i}\left(X, \xi_{i}\right)=-\frac{X(\mathrm{Scal})}{32 n(n+2)} \\
\quad+\frac{1}{2}\left(\omega_{i}\left(\left[\xi_{j}, \xi_{k}\right], X\right)-\omega_{j}\left(\left[\xi_{k}, \xi_{i}\right], X\right)-\omega_{k}\left(\left[\xi_{i}, \xi_{j}\right], X\right)\right), \\
\rho_{i}\left(X, \xi_{j}\right)=\omega_{j}\left(\left[\xi_{j}, \xi_{k}\right], X\right), \quad \rho_{i}\left(X, \xi_{k}\right)=\omega_{k}\left(\left[\xi_{j}, \xi_{k}\right], X\right), \\
\rho_{i}\left(I_{k} X, \xi_{j}\right)=-\quad \rho_{i}\left(I_{j} X, \xi_{k}\right)=g\left(T\left(\xi_{j}, \xi_{k}\right), I_{i} X\right)=\omega_{i}\left(\left[\xi_{j}, \xi_{k}\right], X\right), \\
\quad \rho_{i}\left(\xi_{i}, \xi_{j}\right)+\rho_{k}\left(\xi_{k}, \xi_{j}\right)=\frac{1}{16 n(n+2)} \xi_{j}(\mathrm{Scal}) .
\end{gathered}
$$

The torsion of the Biquard connection restricted to $V$ satisfies the equality

$$
T\left(\xi_{i}, \xi_{j}\right)=-\frac{\text { Scal }}{8 n(n+2)} \xi_{k}-\left[\xi_{i}, \xi_{j}\right]_{H},
$$

where $\left[\xi_{i}, \xi_{j}\right]_{H}$ denotes the projection on $H$ parallel to the vertical space $V$.

We also recall the definition of the $\operatorname{Sp}(n) \operatorname{Sp}(1)$-invariant vector field $A$, which appeared naturally in the Bianchi identities investigated in [IMV]:

$$
A=I_{1}\left[\xi_{2}, \xi_{3}\right]+I_{2}\left[\xi_{3}, \xi_{1}\right]+I_{3}\left[\xi_{1}, \xi_{2}\right]
$$

We shall denote by the same letter the corresponding horizontal 1-form, i.e.,

$$
A(X)=g\left(I_{1}\left[\xi_{2}, \xi_{3}\right]+I_{2}\left[\xi_{3}, \xi_{1}\right]+I_{3}\left[\xi_{1}, \xi_{2}\right], X\right)
$$


The horizontal divergence $\nabla^{*} P$ of a $(0,2)$-tensor field $P$ on $M$ with respect to the Biquard connection is defined to be the $(0,1)$-tensor field

$$
\nabla^{*} P(\cdot)=\left(\nabla_{e_{a}} P\right)\left(e_{a}, \cdot\right)
$$

Then we deduce from [IMV], Theorem 4.8] the following

Theorem 2.6 ([IMV]). On a $(4 n+3)$-dimensional QC manifold with constant qc-scalar curvature we have the formulas

$$
\nabla^{*} T^{0}=(n+2) A, \quad \nabla^{*} U=\frac{1-n}{2} A .
$$

\section{Conformal transformations}

Note that a conformal quaternionic contact transformation between two quaternionic contact manifold is a diffeomorphism $\Phi$ which satisfies

$$
\Phi^{*} \eta=\mu \Psi \cdot \eta
$$

for some positive smooth function $\mu$ and some matrix $\Psi \in S O(3)$ with smooth entries and $\eta$ is an $\mathbb{R}^{3}$-valued 1 -form, $\eta=\left(\eta_{1}, \eta_{2}, \eta_{3}\right)^{t}$ is a column vector with entries 1 -forms. The Biquard connection does not change under rotations, i.e., the Biquard connections of $\Psi \cdot \eta$ and $\eta$ coincide. Hence, studying conformal transformations we may consider only the transformations $\Phi^{*} \eta=\mu \eta$.

Let $h$ be a positive smooth function on a QC manifold $(M, \eta)$. Let $\bar{\eta}=\frac{1}{2 h} \eta$ be a conformal deformation of the QC structure $\eta$. We will denote the objects related to $\bar{\eta}$ by overlining the same object corresponding to $\eta$. Thus, $d \bar{\eta}=-\frac{1}{2 h^{2}} d h \wedge \eta+\frac{1}{2 h} d \eta$ and $\bar{g}=\frac{1}{2 h} g$. The new triple $\left\{\bar{\xi}_{1}, \bar{\xi}_{2}, \bar{\xi}_{3}\right\}$ is determined by the conditions defining the Reeb vector fields. We have

$$
\bar{\xi}_{s}=2 h \xi_{s}+I_{s} \nabla h, \quad s=1,2,3,
$$

where $\nabla h$ is the horizontal gradient defined by $g(\nabla h, X)=d h(X), X \in H$.

The components of the torsion tensor transform according to the following formulas from [IMV], Section 5]:

$$
\begin{aligned}
\bar{T}^{0}(X, Y) & =T^{0}(X, Y)+h^{-1}[\nabla d h]_{[\operatorname{sym}][-1]}(X, Y), \\
\bar{U}(X, Y) & =U(X, Y)+(2 h)^{-1}\left[\nabla d h-2 h^{-1} d h \otimes d h\right]_{[3][0]}(X, Y),
\end{aligned}
$$

where the symmetric part is given by (cf. 3.9)

$$
[\nabla d h]_{[\mathrm{sym}]}(X, Y)=\nabla d h(X, Y)+\sum_{s=1}^{3} d h\left(\xi_{s}\right) \omega_{s}(X, Y)
$$


and [3][0] indicates the trace free part of the [3]-component of the corresponding tensor. In addition, the qc-scalar curvature changes according to the formula [Biq1]

$$
\overline{\mathrm{Scal}}=2 h(\mathrm{Scal})-8(n+2)^{2} h^{-1}|\nabla h|^{2}+8(n+2) \Delta h .
$$

The following vectors will be important for our considerations:

$$
A_{i}=I_{i}\left[\xi_{j}, \xi_{k}\right], \text { hence } A=A_{1}+A_{2}+A_{3} .
$$

Lemma 3.1. Let $h$ be a positive smooth function on a $Q C$ manifold $(M, g, \mathbb{Q})$ with constant qc-scalar curvature Scal $=16 n(n+2)$ and $\bar{\eta}=\frac{1}{2 h} \eta$ a conformal deformation of the qc structure $\eta$. If $\bar{\eta}$ is a 3-Sasakian structure, then

$$
\begin{aligned}
A_{1}(X)= & -\frac{1}{2} h^{-2} d h(X)-\frac{1}{2} h^{-3}|\nabla h|^{2} d h(X)-\frac{1}{2} h^{-1}\left(\nabla d h\left(I_{2} X, \xi_{2}\right)+\nabla d h\left(I_{3} X, \xi_{3}\right)\right) \\
& +\frac{1}{2} h^{-2}\left(d h\left(\xi_{2}\right) d h\left(I_{2} X\right)+d h\left(\xi_{3}\right) d h\left(I_{3} X\right)\right) \\
& +\frac{1}{4} h^{-2}\left(\nabla d h\left(I_{2} X, I_{2} \nabla h\right)+\nabla d h\left(I_{3} X, I_{3} \nabla h\right)\right)
\end{aligned}
$$

The expressions for $A_{2}$ and $A_{3}$ can be obtained from the above formula by a cyclic permutation of $(1,2,3)$. Thus, we also have

$$
\begin{aligned}
A(X)= & -\frac{3}{2} h^{-2} d h(X)-\frac{3}{2} h^{-3}|\nabla h|^{2} d h(X)-h^{-1} \sum_{s=1}^{3} \nabla d h\left(I_{s} X, \xi_{s}\right) \\
& +h^{-2} \sum_{s=1}^{3} d h\left(\xi_{s}\right) d h\left(I_{s} X\right)+\frac{1}{2} h^{-2} \sum_{s=1}^{3} \nabla d h\left(I_{s} X, I_{s} \nabla h\right) .
\end{aligned}
$$

Proof. First we calculate the $\mathrm{sp}(1)$-connection 1-forms of the Biquard connection $\nabla$. For a 3-Sasakian structure we have $\left.d \bar{\eta}_{i}\left(\bar{\xi}_{j}, \bar{\xi}_{k}\right)=2, \bar{\xi}_{i}\right\lrcorner d \bar{\eta}_{i}=0$, the non-zero $\operatorname{sp}(1)$ connection 1-forms are $\bar{\alpha}_{i}\left(\bar{\xi}_{i}\right)=-2, i=1,2,3$, and the qc-scalar curvature $\overline{\text { Scal }}=$ $16 n(n+2)$ (see [IMV], Example 4.12]). Then [3.1], 2.7), and 2.8) yield

$$
\begin{gathered}
2 d \eta_{i}\left(\xi_{j}, \xi_{k}\right)=2 h^{-1}+h^{-2}\|d h\|^{2}, \quad \alpha_{i}(X)=-h^{-1} d h\left(I_{i} X\right), \\
\alpha_{i}\left(\xi_{j}\right)=-h^{-1} d h\left(\xi_{k}\right)=-\alpha_{j}\left(\xi_{i}\right), \quad 4 \alpha_{i}\left(\xi_{i}\right)=-4-2 h^{-1}-h^{-2}\|d h\|^{2} .
\end{gathered}
$$

From the 3-Sasakian assumption the commutators are $\left[\bar{\xi}_{i}, \bar{\xi}_{j}\right]=-2 \bar{\xi}_{k}$. Thus, for $X \in H$ taking also into account 3.11 we have

$$
g\left(\left[\bar{\xi}_{1}, \bar{\xi}_{2}\right], I_{3} X\right)=-2 g\left(\bar{\xi}_{3}, I_{3} X\right)=-2 g\left(2 h \xi_{3}+I_{3} \nabla h, I_{3} X\right)=-2 d h(X) .
$$

Therefore, using again 3.1, we obtain

$$
\begin{aligned}
-2 d h(X)= & g\left(\left[\bar{\xi}_{1}, \bar{\xi}_{2}\right], I_{3} X\right)=g\left(\left[2 h \xi_{1}+I_{1} \nabla h, 2 h \xi_{2}+I_{2} \nabla h\right], I_{3} X\right) \\
= & -4 h^{2} A_{3}(X)+2 h g\left(\left[\xi_{1}, I_{2} \nabla h\right], I_{3} X\right)+2 h g\left(\left[I_{1} \nabla h, \xi_{2}\right], I_{3} X\right) \\
& +g\left(\left[I_{1} \nabla h, I_{2} \nabla h\right], I_{3} X\right) .
\end{aligned}
$$


The last three terms are transformed as follows. The first equals

$$
\begin{aligned}
g\left(\left[\xi_{1}, I_{2} \nabla h\right], I_{3} X\right)= & g\left(\left(\nabla_{\xi_{1}} I_{2}\right) \nabla h+I_{2} \nabla_{\xi_{1}} \nabla h, I_{3} X\right)-g\left(T\left(\xi_{1}, I_{2} \nabla h\right), I_{3} X\right) \\
= & -\alpha_{3}\left(\xi_{1}\right) d h\left(I_{2} X\right)+\alpha_{1}\left(\xi_{1}\right) d h(X)-\nabla d h\left(\xi_{1}, I_{1} X\right) \\
& -g\left(T\left(\xi_{1}, I_{2} \nabla h\right), I_{3} X\right),
\end{aligned}
$$

where we use (2.6) and the fact that $\nabla$ preserves the splitting $H \oplus V$. The second term is

$$
\begin{aligned}
g\left(\left[I_{1} \nabla h, \xi_{2}\right], I_{3} X\right)= & \alpha_{2}\left(\xi_{2}\right) d h(X)+\alpha_{3}\left(\xi_{2}\right) d h\left(I_{1} X\right)-\nabla d h\left(\xi_{2}, I_{2} X\right) \\
& -g\left(T\left(I_{1} \nabla h, \xi_{2}\right), I_{3} X\right)
\end{aligned}
$$

and finally

$$
\begin{aligned}
g\left(\left[I_{1} \nabla h, I_{2} \nabla h\right], I_{3} X\right)= & -\alpha_{3}\left(I_{1} \nabla h\right) d h\left(I_{2} X\right)+\alpha_{1}\left(I_{1} \nabla h\right) d h(X)-\nabla d h\left(I_{1} \nabla h, I_{1} X\right) \\
& +\alpha_{2}\left(I_{2} \nabla h\right) d h(X)+\alpha_{3}\left(I_{2} \nabla h\right) d h\left(I_{1} X\right)-\nabla d h\left(I_{2} \nabla h, I_{2} X\right) .
\end{aligned}
$$

Next we apply (3.7) to the last three equalities, then substitute their sum into (3.8), after which we use the commutation relations

$$
\begin{aligned}
& \nabla d h(X, Y)-\nabla d h(Y, X)=-d h(T(X, Y))=-2 \sum_{s=1}^{3} \omega_{S}(X, Y) d h\left(\xi_{s}\right), \\
& \nabla d h(X, \xi)-\nabla d h(\xi, X)=-d h(T(X, \xi)), \quad X, Y \in H, \xi \in V .
\end{aligned}
$$

The result is the following identity:

$$
\begin{aligned}
4 h^{2} A_{3}(X)= & \left(-4 h+h^{-1}\|\nabla h\|^{2}\right) d h(X)-2 h\left[\nabla d h\left(I_{1} X, \xi_{1}\right)+\nabla d h\left(I_{2} X, \xi_{2}\right)\right] \\
& -\left[\nabla d h\left(I_{1} X, I_{1} \nabla h\right)+\nabla d h\left(I_{2} X, I_{2} \nabla h\right)\right] \\
& +2\left[d h\left(\xi_{1}\right) d h\left(I_{1} X\right)+d h\left(\xi_{2}\right) d h\left(I_{2} X\right)+2 d h\left(\xi_{3}\right) d h\left(I_{3} X\right)\right] \\
& +2 h\left[T\left(\xi_{1}, I_{1} X, \nabla h\right)+T\left(\xi_{2}, I_{2} X, \nabla h\right)-T\left(\xi_{1}, I_{2} X, I_{3} \nabla h\right)\right. \\
& \left.+T\left(\xi_{2}, I_{1} X, I_{3} \nabla h\right)\right],
\end{aligned}
$$

where $T(\xi, X, Y)=g\left(T_{\xi} X, Y\right)$ for a vertical vector $\xi$ and horizontal vectors $X$ and $Y$. With the help of Proposition 2.3 we decompose the torsions into symmetric and antisymmetric part $T_{\xi_{i}}=T_{\xi_{i}}^{0}+I_{i} U, i=1,2,3$, and then express the symmetric parts of the torsion terms in the form $T_{\xi_{1}}^{0}=\left(T_{\xi_{1}}^{0}\right)^{--+}+\left(T_{\xi_{1}}^{0}\right)^{-+-}, T_{\xi_{2}}^{0}=\left(T_{\xi_{2}}^{0}\right)^{--+}+$ $\left(T_{\xi_{2}}^{0}\right)^{+--}$. Hence, using $T^{0^{--+}}=2\left(T_{\xi_{2}}^{0}\right)^{+--} I_{2}=2\left(T_{\xi_{1}}^{0}\right)^{-+-} I_{1}$ etc., which follows again from Proposition 2.3, the sum of the torsion terms in 3.10 can be seen to equal $2\left(T^{0}\right)^{--+}(X, \nabla h)-4 U(X, \nabla h)$. This allows us to rewrite 3.10 in the form

$$
\begin{aligned}
4 A_{3}(X)= & \left(-4 h^{-1}+h^{-3}\|\nabla h\|^{2}\right) d h(X)-2 h^{-1}\left[\nabla d h\left(I_{1} X, \xi_{1}\right)+\nabla d h\left(I_{2} X, \xi_{2}\right)\right] \\
& +2 h^{-2}\left[d h\left(\xi_{1}\right) d h\left(I_{1} X\right)+d h\left(\xi_{2}\right) d h\left(I_{2} X\right)+2 d h\left(\xi_{3}\right) d h\left(I_{3} X\right)\right] \\
& -h^{-2}\left[\nabla d h\left(I_{1} X, I_{1} \nabla h\right)+\nabla d h\left(I_{2} X, I_{2} \nabla h\right)\right] \\
& +4 h^{-1}\left[\left(T^{0}\right)^{--+}(\nabla h, X)-2 U(\nabla h, X)\right] .
\end{aligned}
$$

Using (3.2) the $\left(T^{0}\right)^{--+}$component of the torsion can be expressed by $h$ as follows (see (2.1) and (2.9): 
$4\left(T^{0}\right)^{--+}(\nabla h, X)=T^{0}(\nabla h, X)-T^{0}\left(I_{1} \nabla h, I_{1} X\right)-T^{0}\left(I_{2} \nabla h, I_{2} X\right)+T^{0}\left(I_{3} \nabla h, I_{3} X\right)$

$=-h^{-1}\left\{[\nabla d h]_{[-1]}(\nabla h, X)-[\nabla d h]_{[-1]}\left(I_{1} \nabla h, I_{1} X\right)-[\nabla d h]_{[-1]}\left(I_{2} \nabla h, I_{2} X\right)\right.$ $\left.+[\nabla d h]_{[-1]}\left(I_{3} \nabla h, I_{3} X\right)\right\}$

$-h^{-1} \sum_{s=1}^{3}\left\{d h\left(\xi_{s}\right)\left[g\left(I_{s} \nabla h, X\right)-g\left(I_{s} I_{1} \nabla h, I_{1} X\right)-g\left(I_{s} I_{2} \nabla h, I_{2} X\right)+g\left(I_{s} I_{3} \nabla h, I_{3} X\right)\right]\right\}$

$=-h^{-1}\left\{\nabla d h(\nabla h, X)-\nabla d h\left(I_{1} \nabla h, I_{1} X\right)-\nabla d h\left(I_{2} \nabla h, I_{2} X\right)+\nabla d h\left(I_{3} \nabla h, I_{3} X\right)\right\}$

$+4 h^{-1} d h\left(\xi_{3}\right) d h\left(I_{3} X\right)$.

Invoking equation 3.9 we can put $\nabla h$ in the second place in the Hessian terms, thus, proving the formula

$$
\begin{aligned}
& 4\left(T^{0}\right)^{--+}(\nabla h, X)=-4 h^{-1} d h\left(\xi_{3}\right) d h\left(I_{3} X\right) \\
& -h^{-1}\left\{\nabla d h(X, \nabla h)-\nabla d h\left(I_{1} X, I_{1} \nabla h\right)-\nabla d h\left(I_{2} X, I_{2} \nabla h\right)+\nabla d h\left(I_{3} X, I_{3} \nabla h\right)\right\} .
\end{aligned}
$$

On the other hand, 2.10, 3.3 and the Yamabe equation 3.4 give

$$
\begin{aligned}
& 8 U(\nabla h, X)=-h^{-1}\left\{\nabla d h(\nabla h, X)+\sum_{s=1}^{3} \nabla d h\left(I_{s} \nabla h, I_{s} X\right)\right. \\
& \left.-2 h^{-1}\|\nabla h\|^{2} d h(X)-\frac{\triangle h}{n} d h(X)+2 h^{-1} \frac{\|\nabla h\|^{2}}{n} d h(X)\right\} \\
& =-h^{-1}\left\{\nabla d h(\nabla h, X)+\sum_{s=1}^{3} \nabla d h\left(I_{s} \nabla h, I_{s} X\right)\right\} \\
& -h^{-1}\left\{-2 h^{-1}\|\nabla h\|^{2} d h(X)-\frac{2 n-4 n h+(n+2) h^{-1}\|\nabla h\|^{2}}{n} d h(X)+2 h^{-1} \frac{\|\nabla h\|^{2}}{n} d h(X)\right\} \\
& =-h^{-1}\left\{\nabla d h(X, \nabla h)+\sum_{s=1}^{3} \nabla d h\left(I_{s} X, I_{s} \nabla h\right)\right\}-h^{-1}\left(-3 h^{-1}\|\nabla h\|^{2}-2+4 h\right) d h(X) \text {. }
\end{aligned}
$$

Substituting the last two formulas in 3.11 gives $A_{3}$ in the form of (3.6) written for $A_{1}$ (cf. the paragraph after 3.6).

\section{Divergence formulas}

We shall need the divergences of various vectors/forms through the almost complex structures, so we start with a general formula valid for any horizontal vector/form $A$. Let $\left\{e_{1}, \ldots, e_{4 n}\right\}$ be an orthonormal basis of $H$. The divergence of $I_{1} A$ is

$$
\nabla^{*}\left(I_{1} A\right) \equiv\left(\nabla_{e_{a}}\left(I_{1} A\right)\right)\left(e_{a}\right)=-\left(\nabla_{e_{a}} A\right)\left(I_{1} e_{a}\right)-A\left(\left(\nabla_{e_{a}} I_{1}\right) e_{a}\right),
$$

recalling $I_{1} A(X)=-A\left(I_{1} X\right)$. 
We say that an orthonormal frame

$$
\left\{e_{1}, e_{2}=I_{1} e_{1}, e_{3}=I_{2} e_{1}, e_{4}=I_{3} e_{1}, \ldots, e_{4 n}=I_{3} e_{4 n-3}, \xi_{1}, \xi_{2}, \xi_{3}\right\}
$$

is a qc-normal frame (at a point) if the connection 1-forms of the Biquard connection vanish (at that point). Lemma 4.5 in [IMV] asserts that a qc-normal frame exists at each point of a QC manifold. With respect to a qc-normal frame the above divergence reduces to

$$
\nabla^{*}\left(I_{1} A\right)=-\left(\nabla_{e_{a}} A\right)\left(I_{1} e_{a}\right)
$$

Lemma 4.1. Suppose $(M, \eta, \mathbb{Q})$ is a quaternionic contact manifold with constant $q c$ scalar curvature. For any function $h$ we have

$$
\begin{aligned}
& \nabla^{*}\left(\sum_{s=1}^{3} d h\left(\xi_{s}\right) I_{s} A_{s}\right)=\sum_{s=1}^{3} \nabla d h\left(I_{s} e_{a}, \xi_{s}\right) A_{s}\left(e_{a}\right), \\
& \nabla^{*}\left(\sum_{s=1}^{3} d h\left(\xi_{s}\right) I_{s} A\right)=\sum_{s=1}^{3} \nabla d h\left(I_{s} e_{a}, \xi_{s}\right) A\left(e_{a}\right) .
\end{aligned}
$$

Proof. Using the identification of the 3-dimensional vector spaces spanned by $\left\{\xi_{1}, \xi_{2}, \xi_{3}\right\}$ and $\left\{I_{1}, I_{2}, I_{3}\right\}$ with $\mathbb{R}^{3}$, the restriction of the action of $\operatorname{Sp}(n) \operatorname{Sp}(1)$ to these spaces can be identified with the action of the group $S O(3)$, i.e., $\xi_{i}=\sum_{t=1}^{3} \Psi_{i t} \bar{\xi}_{t}$ and $I_{i}=\sum_{t=1}^{3} \Psi_{i t} \bar{I}_{t}, i=1,2,3$, with $\Psi \in S O(3)$. One verifies easily that the vectors $A$, $\sum_{s=1}^{3} d h\left(\xi_{s}\right) I_{s} A_{s}=-\sum_{i=1}^{3} d h\left(\xi_{i}\right)\left[\xi_{j}, \xi_{k}\right]$ and $\sum_{s=1}^{3} d h\left(\xi_{s}\right) I_{s} A$ are $\operatorname{Sp}(n) \operatorname{Sp}(1)$ invariant on $\mathbb{H}$, for example $A=(\operatorname{det} \Psi) A$. Thus, it is sufficient to compute their divergences in a qc-normal frame. To avoid the introduction of new variables, in this proof, we shall assume that $\left\{e_{1}, \ldots, e_{4 n}, \xi_{1}, \xi_{2}, \xi_{3}\right\}$ is a qc-normal frame.

We apply (2.14). Using that the Biquard connection preserves the splitting of $T M$, we find

$$
\begin{aligned}
\nabla^{*}\left[\xi_{1}, \xi_{2}\right] & =-g\left(\nabla_{e_{a}}\left(T\left(\xi_{1}, \xi_{2}\right)\right), e_{a}\right) \\
& =-g\left(\left(\nabla_{e_{a}} T\right)\left(\xi_{1}, \xi_{2}\right), e_{a}\right)-g\left(T\left(\nabla_{e_{a}} \xi_{1}, \xi_{2}\right), e_{a}\right)-g\left(T\left(\xi_{1}, \nabla_{e_{a}} \xi_{2}\right), e_{a}\right)
\end{aligned}
$$

From Bianchi's identity we have $\left(\sigma_{A, B, C}\right.$ means a cyclic sum over $\left.(A, B, C)\right)$

$$
\begin{aligned}
g\left(\left(\nabla_{e_{a}} T\right)\left(\xi_{1}, \xi_{2}\right), e_{a}\right) & =-g\left(\left(\nabla_{\xi_{1}} T\right)\left(\xi_{2}, e_{a}\right), e_{a}\right)-g\left(\left(\nabla_{\xi_{2}} T\right)\left(e_{a}, \xi_{1}\right), e_{a}\right) \\
& -g\left(\sigma_{e_{a}, \xi_{1}, \xi_{2}}\left\{T\left(T\left(e_{a}, \xi_{1}\right), \xi_{2}\right)\right\}, e_{a}\right)+g\left(\sigma_{e_{a}, \xi_{1}, \xi_{2}}\left\{R\left(e_{a}, \xi_{1}\right) \xi_{2}\right\}, e_{a}\right) \\
= & -g\left(T\left(T\left(e_{a}, \xi_{1}\right), \xi_{2}\right), e_{a}\right)-g\left(T\left(T\left(\xi_{1}, \xi_{2}\right), e_{a}\right), e_{a}\right)-g\left(T\left(T\left(\xi_{2}, e_{a}\right), \xi_{1}\right), e_{a}\right) \\
= & g\left(T\left(T\left(\xi_{1}, e_{a}\right), \xi_{2}\right), e_{a}\right)-g\left(T\left(T\left(\xi_{2}, e_{a}\right), \xi_{1}\right), e_{a}\right)-g\left(T\left(T\left(\xi_{1}, \xi_{2}\right), e_{a}\right), e_{a}\right),
\end{aligned}
$$

taking into account that as mappings on $H$ the torsion tensors $T\left(\xi_{i}, X\right)$ and the curvature tensor $R\left(\xi_{1}, \xi_{2}\right)$ are traceless, so $g\left(\left(\nabla_{\xi_{1}} T\right)\left(\xi_{2}, e_{a}\right), e_{a}\right)=0$ and $g\left(R\left(\xi_{1}, \xi_{2}\right) e_{a}, e_{a}\right)=0$, while the connection preserves the splitting, to obtain the next to last line. The last term 
is equal to zero as

$$
\begin{aligned}
g\left(T\left(T\left(\xi_{1}, \xi_{2}\right), e_{a}\right), e_{a}\right) & =g\left(T\left(-\frac{\mathrm{Scal}}{8 n(n+2)} \xi_{3}-\left[\xi_{1}, \xi_{2}\right]_{H}, e_{a}\right), e_{a}\right) \\
& =-\frac{\mathrm{Scal}}{8 n(n+2)} g\left(T\left(\xi_{3}, e_{a}\right), e_{a}\right)=0,
\end{aligned}
$$

taking into account that the torsion $T_{\xi_{3}}$ is traceless and $T\left(\left[\xi_{1}, \xi_{2}\right]_{H}, e_{a}\right)$ is a vertical vector. On the other hand,

$$
\begin{aligned}
g\left(T\left(T_{\xi_{1}} e_{a}, \xi_{2}\right), e_{a}\right)-g\left(T\left(T_{\xi_{2}} e_{a}, \xi_{1}\right), e_{a}\right) & \\
= & -\left[g\left(T\left(e_{b}, \xi_{2}\right), e_{a}\right) g\left(T\left(\xi_{1}, e_{a}\right), e_{b}\right)-g\left(T\left(e_{b}, \xi_{1}\right), e_{a}\right) g\left(T\left(\xi_{2}, e_{a}\right), e_{b}\right)\right] \\
& =\left[g\left(T\left(\xi_{2}, e_{b}\right), e_{a}\right) g\left(T\left(\xi_{1}, e_{a}\right), e_{b}\right)-g\left(T\left(\xi_{1}, e_{b}\right), e_{a}\right) g\left(T\left(\xi_{2}, e_{a}\right), e_{b}\right)\right]=0 .
\end{aligned}
$$

The equalities $\nabla^{*}\left(I_{1} A_{1}\right)=\nabla^{*}\left(I_{2} A_{2}\right)=0$ with respect to a qc-normal frame can be obtained similarly. Hence, the first formula in Lemma 4.1 follows.

We are left with proving the second divergence formula. Since the scalar curvature is constant, 2.12) implies

$$
A(X)=-2 \sum_{s=1}^{3} \rho_{s}\left(X, \xi_{s}\right) .
$$

Fix an $s \in\{1,2,3\}$. Working again in a qc-normal frame we have

$$
\left(\nabla_{e_{a}} A\right)\left(I_{s} e_{a}\right)=-2 \sum_{t=1}^{3}\left(\nabla_{e_{a}} \rho_{t}\right)\left(I_{s} e_{a}, \xi_{t}\right)
$$

A calculation involving the expressions 2.11 and the properties of the torsion shows that

$$
\operatorname{tr}\left(\rho_{t} \circ I_{s}\right)=-\frac{1}{2(n+2)} \delta_{s t} \text { Scal } .
$$

The second Bianchi identity

$$
\begin{aligned}
0= & g\left(\left(\nabla_{e_{a}} R\right)\left(I_{s} e_{a}, \xi_{t}\right) e_{b}, I_{t} e_{b}\right)+g\left(\left(\nabla_{I_{s} e_{a}} R\right)\left(\xi_{t}, e_{a}\right) e_{b}, I_{t} e_{b}\right) \\
& +g\left(\left(\nabla_{\xi_{t}} R\right)\left(e_{a}, I_{s} e_{a}\right) e_{b}, I_{t} e_{b}\right)+g\left(R\left(T\left(e_{a}, I_{s} e_{a}\right), \xi_{t}\right) e_{b}, I_{t} e_{b}\right) \\
& +g\left(R\left(T\left(I_{s} e_{a}, \xi_{t}\right), e_{a}\right) e_{b}, I_{t} e_{b}\right)+g\left(R\left(T\left(\xi_{t}, e_{a}\right), I_{s} e_{a}\right) e_{b}, I_{t} e_{b}\right)
\end{aligned}
$$

together with the constancy of the qc-scalar curvature and (4.2) show that the third term on the right is zero and thus

$$
\sum_{t=1}^{3}\left\{2\left(\nabla_{e_{a}} \rho_{t}\right)\left(I_{s} e_{a}, \xi_{t}\right)-2 \rho_{t}\left(T\left(\xi_{t}, I_{s} e_{a}\right), e_{a}\right)+\rho_{t}\left(T\left(e_{a}, I_{s} e_{a}\right), \xi_{t}\right)\right\}=0 .
$$

Substituting 2.5) in the above equality we come to the equation

$$
\sum_{t=1}^{3}\left(\nabla_{e_{a}} \rho_{t}\right)\left(I_{s} e_{a}, \xi_{t}\right)=\sum_{t=1}^{3} \rho_{t}\left(T\left(\xi_{t}, I_{s} e_{a}\right), e_{a}\right)-4 n \sum_{t=1}^{3} \rho_{t}\left(\xi_{s}, \xi_{t}\right)=0
$$

where the vanishing of the second term follows from 2.13 , while the vanishing of the 
first term is seen as follows. Using the standard inner product on $\operatorname{End}(H)$

$$
g(C, B)=\operatorname{tr}\left(B^{*} C\right)=\sum_{a=1}^{4 n} g\left(C\left(e_{a}\right), B\left(e_{a}\right)\right),
$$

where $C, B \in \operatorname{End}(H),\left\{e_{1}, \ldots, e_{4 n}\right\}$ is a $g$-orthonormal basis of $H$, the definition of $T_{\xi_{s}}^{0}$, the formulas in Theorem 2.5 and Proposition 2.3 imply

$$
\begin{aligned}
\sum_{s=1}^{3} \rho_{s}( & \left.T\left(\xi_{s}, I_{1} e_{a}\right), e_{a}\right) \\
= & g\left(\rho_{1}, T_{\xi_{1}}^{0} I_{1}\right)+g\left(\rho_{2}, T_{\xi_{2}}^{0} I_{1}\right)+g\left(\rho_{3}, T_{\xi_{3}}^{0} I_{1}\right)-g\left(\rho_{1}, u\right)-g\left(\rho_{2}, I_{3} u\right)+g\left(\rho_{3}, I_{2} u\right) \\
= & g\left(\rho_{1}, T_{\xi_{1}}^{0} I_{1}\right)+g\left(\rho_{2}, T_{\xi_{2}}^{0} I_{1}\right)+g\left(\rho_{3}, T_{\xi_{3}}^{0} I_{1}\right) \\
= & g\left(2\left(T_{\xi_{2}}^{0}\right)^{--+} I_{3}-2 I_{1} u-\frac{\mathrm{Scal}}{8 n(n+2)} I_{1}, T_{\xi_{1}}^{0} I_{1}\right) \\
& +g\left(2\left(T_{\xi_{3}}^{0}\right)^{+--} I_{1}-2 I_{2} u-\frac{\mathrm{Scal}}{8 n(n+2)} I_{2}, T_{\xi_{2}}^{0} I_{1}\right) \\
& +g\left(2\left(T_{\xi_{1}}^{0}\right)^{-+-} I_{2}-2 I_{3} u-\frac{\mathrm{Scal}}{8 n(n+2)} I_{3}, T_{\xi_{3}}^{0} I_{1}\right) \\
= & -2 g\left(\left(T_{\xi_{2}}^{0}\right)^{--+} I_{2}, T_{\xi_{1}}^{0}\right)+2 g\left(\left(T_{\xi_{3}}^{0}\right)^{+--}, T_{\xi_{2}}^{0}\right)+2 g\left(\left(T_{\xi_{1}}^{0}\right)^{-+-} I_{3}, T_{\xi_{3}}^{0}\right) \\
= & 2 g\left(\left(T_{\xi_{3}}^{0}\right)^{+--},\left(T_{\xi_{2}}^{0}\right)^{+--}\right)+2 g\left(\left(T_{\xi_{1}}^{0}\right)^{-+-}, I_{3}\left(T_{\xi_{3}}^{0}\right)^{+--}\right) \\
= & 2 g\left(I_{2}\left(T_{\xi_{3}}^{0}\right)^{+--}, I_{2}\left(T_{\xi_{2}}^{0}\right)^{+--}\right)-2 g\left(I_{1}\left(T_{\xi_{1}}^{0}\right)^{-+-}, I_{2}\left(T_{\xi_{3}}^{0}\right)^{+--}\right)=0 .
\end{aligned}
$$

Renaming the almost complex structures shows that the same conclusion is true when we replace $I_{1}$ with $I_{2}$ or $I_{3}$ in the above calculation.

Finally, the second formula in Lemma 4.1 follows from (4.1) and (4.3).

We shall also need the following 1-forms:

$$
\begin{aligned}
& D_{1}(X)=-h^{-1}\left(T^{0}\right)^{+--}(X, \nabla h), \\
& D_{2}(X)=-h^{-1}\left(T^{0}\right)^{-+-}(X, \nabla h), \\
& D_{3}(X)=-h^{-1}\left(T^{0}\right)^{--+}(X, \nabla h) .
\end{aligned}
$$

For simplicity, using the musical isomorphism, we will denote by $D_{1}, D_{2}, D_{3}$ the corresponding (horizontal) vector fields, for example $g\left(D_{1}, X\right)=D_{1}(X)$ for $X \in H$. Finally, we set

$$
D=D_{1}+D_{2}+D_{3}=-h^{-1} T^{0}(X, \nabla h) .
$$

Lemma 4.2. Suppose $(M, \eta)$ is a quaternionic contact manifold with constant qc-scalar curvature Scal $=16 n(n+2)$. Suppose $\bar{\eta}=\frac{1}{2 h} \eta$ has vanishing $[-1]$-torsion component $\bar{T}^{0}=0$. Then

$$
D(X)=\frac{1}{4} h^{-2}\left(3 \nabla d h(X, \nabla h)-\sum_{s=1}^{3} \nabla d h\left(I_{s} X, I_{s} \nabla h\right)\right)+h^{-2} \sum_{s=1}^{3} d h\left(\xi_{s}\right) d h\left(I_{s} X\right) .
$$


and the divergence of $D$ satisfies

$$
\nabla^{*} D=\left|T^{0}\right|^{2}-h^{-1} g(d h, D)-h^{-1}(n+2) g(d h, A) .
$$

Proof. (a) The formula for $D$ follows immediately from 3.2.

(b) We work in a qc-normal frame. Since the scalar curvature is assumed to be constant we use 2.15 ) to find

$$
\begin{aligned}
\nabla^{*} D & =-h^{-1} d h\left(e_{a}\right) D\left(e_{a}\right)-h^{-1} \nabla^{*} T^{0}(\nabla h)-h^{-1} T^{0}\left(e_{a}, e_{b}\right) \nabla d h\left(e_{a}, e_{b}\right) \\
& =-h^{-1} d h\left(e_{a}\right) D\left(e_{a}\right)-h^{-1}(n+2) d h\left(e_{a}\right) A\left(e_{a}\right)-g\left(T^{0}, h^{-1} \nabla d h\right) \\
& =\left|T^{0}\right|^{2}-h^{-1} d h\left(e_{a}\right) D\left(e_{a}\right)-h^{-1}(n+2) d h\left(e_{a}\right) A\left(e_{a}\right),
\end{aligned}
$$

using (3.2) in the last equality.

Let us also consider the following 1-forms (and corresponding vectors):

$$
F_{s}(X)=-h^{-1} T^{0}\left(X, I_{s} \nabla h\right), \quad X \in H, s=1,2,3 .
$$

From the definition of $F_{1}$ and (4.4) we find

$$
\begin{aligned}
F_{1}(X) & =-h^{-1} T^{0}\left(X, I_{1} \nabla h\right) \\
& =-h^{-1}\left(T^{0}\right)^{+--}\left(X, I_{1} \nabla h\right)-h^{-1}\left(T^{0}\right)^{-+-}\left(X, I_{1} \nabla h\right)-h^{-1}\left(T^{0}\right)^{--+}\left(X, I_{1} \nabla h\right) \\
& =h^{-1}\left(T^{0}\right)^{+--}\left(I_{1} X, \nabla h\right)-h^{-1}\left(T^{0}\right)^{-+-}\left(I_{1} X, \nabla h\right)-h^{-1}\left(T^{0}\right)^{--+}\left(I_{1} X, \nabla h\right) \\
& =-D_{1}\left(I_{1} X\right)+D_{2}\left(I_{1} X\right)+D_{3}\left(I_{1} X\right) .
\end{aligned}
$$

Thus, the forms $F_{s}$ can be expressed by the forms $D_{s}$ as follows:

$$
\begin{aligned}
& F_{1}(X)=-D_{1}\left(I_{1} X\right)+D_{2}\left(I_{1} X\right)+D_{3}\left(I_{1} X\right), \\
& F_{2}(X)=D_{1}\left(I_{2} X\right)-D_{2}\left(I_{2} X\right)+D_{3}\left(I_{2} X\right), \\
& F_{3}(X)=D_{1}\left(I_{3} X\right)+D_{2}\left(I_{3} X\right)-D_{3}\left(I_{3} X\right) .
\end{aligned}
$$

Lemma 4.3. Suppose $(M, \eta)$ is a quaternionic contact manifold with constant qc-scalar curvature Scal $=16 n(n+2)$. Suppose $\bar{\eta}=\frac{1}{2 h} \eta$ has vanishing $[-1]$-torsion component, $\bar{T}^{0}=0$. Then

$$
\begin{aligned}
\nabla^{*}\left(\sum_{s=1}^{3} d h\left(\xi_{s}\right) F_{s}\right)= & \sum_{s=1}^{3}\left[\nabla d h\left(I_{s} e_{a}, \xi_{s}\right) F_{s}\left(I_{s} e_{a}\right)\right] \\
& +h^{-1} \sum_{s=1}^{3}\left[d h\left(\xi_{s}\right) d h\left(I_{s} e_{a}\right) D\left(e_{a}\right)+(n+2) d h\left(\xi_{s}\right) d h\left(I_{s} e_{a}\right) A\left(e_{a}\right)\right]
\end{aligned}
$$

Proof. We note that the vector $\sum_{s=1}^{3} d h\left(\xi_{s}\right) F_{s}$ is an $\operatorname{Sp}(n) \operatorname{Sp}(1)$-invariant vector, hence we may assume that $\left\{e_{1}, \ldots, e_{4 n}, \xi_{1}, \xi_{2}, \xi_{3}\right\}$ is a qc-normal frame. Since the scalar curvature is assumed to be constant we can apply Theorem 2.6, thus $\nabla^{*} T^{0}=(n+2) A$. Turning to the divergence, we compute 


$$
\begin{aligned}
& \nabla^{*}\left(\sum_{s=1}^{3} d h\left(\xi_{s}\right) F_{s}\right)=\sum_{s=1}^{3}\left[\nabla d h\left(e_{a}, \xi_{s}\right) F_{s}\left(e_{a}\right)\right]-\sum_{s=1}^{3} h^{-1} d h\left(\xi_{s}\right) \nabla^{*} T^{0}\left(I_{s} \nabla h\right) \\
& \quad+\sum_{s=1}^{3}\left[h^{-2} d h\left(\xi_{s}\right) d h\left(e_{a}\right) T^{0}\left(e_{a}, I_{s} e_{b}\right) d h\left(e_{b}\right)-h^{-1} d h\left(\xi_{s}\right) T^{0}\left(e_{a}, I_{s} e_{b}\right) \nabla d h\left(e_{a}, e_{b}\right)\right] \\
& =\sum_{s=1}^{3}\left[\nabla d h\left(e_{a}, \xi_{s}\right) F_{s}\left(e_{a}\right)\right]-\sum_{s=1}^{3} h^{-1} d h\left(\xi_{s}\right) \nabla^{*} T^{0}\left(I_{s} \nabla h\right) \\
& \quad+\sum_{s=1}^{3}\left[h^{-1} d h\left(\xi_{s}\right) d h\left(I_{s} e_{a}\right) D\left(e_{a}\right)\right] \\
& =\sum_{s=1}^{3}\left[\nabla d h\left(e_{a}, \xi_{s}\right) F_{s}\left(e_{a}\right)+h^{-1} d h\left(\xi_{s}\right) d h\left(I_{s} e_{a}\right) D\left(e_{a}\right)\right. \\
& \left.\quad+h^{-1}(n+2) d h\left(\xi_{s}\right) d h\left(I_{s} e_{a}\right) A\left(e_{a}\right)\right]
\end{aligned}
$$

using the symmetry of $T^{0}$ in the next to last equality and the fact $T^{0}\left(e_{a}, I_{1} e_{b}\right) \nabla d h\left(e_{a}, e_{b}\right)$ $=0$. The latter can be seen, for example, by first using (3.2) and the formula for the symmetric part of $\nabla d h$ given after 3.3 from which we have

$$
\begin{array}{rl}
T^{0}\left(e_{a}, I_{1} e_{b}\right) \nabla d & h\left(e_{a}, e_{b}\right) \\
= & -h^{-1} \nabla d h_{[\mathrm{sym}][-1]}\left(e_{a}, I_{1} e_{b}\right)\left[\nabla d h_{[\mathrm{sym}]}\left(e_{a}, e_{b}\right)-\sum_{s=1}^{3} d h\left(\xi_{s}\right) \omega_{s}\left(e_{a}, e_{b}\right)\right] \\
= & -h^{-1} \nabla d h_{[\mathrm{sym}][-1]}\left(e_{a}, I_{1} e_{b}\right) \nabla d h_{[\mathrm{sym}][-1]}\left(e_{a}, e_{b}\right) \\
& -h^{-1} \nabla d h_{[\mathrm{sym}][-1]}\left(e_{a}, I_{1} e_{b}\right) \nabla d h_{[\mathrm{sym}][3]}\left(e_{a}, e_{b}\right) \\
& +h^{-1} \nabla d h_{[\mathrm{sym}][-1]}\left(e_{a}, I_{1} e_{b}\right) \sum_{s=1}^{3} d h\left(\xi_{s}\right) \omega_{s}\left(e_{a}, e_{b}\right)=0,
\end{array}
$$

using the zero traces of the [-1]-component to justify the vanishing of the third term in the last equality. Switching to the basis $\left\{I_{s} e_{a}: a=1, \ldots, 4 n\right\}$ in the first term of the right-hand side of (4.7) completes the proof.

At this point we restrict our considerations to the 7-dimensional case, i.e. $n=1$. Following is our main technical result. As mentioned in the introduction, we were motivated to seek a divergence formula of this type based on the Riemannian and CR cases of the problem in question. The main difficulty was to find a suitable vector field with non-negative divergence containing the norm of the torsion. The fulfilment of this task was facilitated by the results of [IMV], which in particular showed that similarly to the CR case, but unlike the Riemannian case, we were not able to achieve a proof based purely on the Bianchi identities (see [IMV], Theorem 4.8]).

Theorem 4.4. Suppose $\left(M^{7}, \eta\right)$ is a quaternionic contact structure conformal to a 3Sasakian structure $\left(M^{7}, \bar{\eta}\right), \tilde{\eta}=\frac{1}{2 h} \eta$. If $\operatorname{Scal}_{\eta}=\operatorname{Scal}_{\tilde{\eta}}=16 n(n+2)$, then with $f$ given by 
the following identity holds:

$$
f=\frac{1}{2}+h+\frac{1}{4} h^{-1}|\nabla h|^{2},
$$

$\nabla^{*}\left(f D+\sum_{s=1}^{3} d h\left(\xi_{s}\right) F_{s}+4 \sum_{s=1}^{3} d h\left(\xi_{s}\right) I_{s} A_{s}-\frac{10}{3} \sum_{s=1}^{3} d h\left(\xi_{s}\right) I_{s} A\right)=f\left|T^{0}\right|^{2}+h\langle Q V, V\rangle$.

Here, $Q$ is a positive semi-definite matrix and $V=\left(D_{1}, D_{2}, D_{3}, A_{1}, A_{2}, A_{3}\right)$ with $A_{s}$, $D_{s}$ defined, respectively, in (3.5) and 4.4.

Proof. Using the formulas for the divergences of $D, \sum_{s=1}^{3} d h\left(\xi_{s}\right) F_{s}, \sum_{s=1}^{3} d h\left(\xi_{s}\right) I_{s} A_{s}$ and $\sum_{s=1}^{3} d h\left(\xi_{s}\right) I_{s} A$ given respectively in Lemmas 4.2 , 4.3 and 4.1 we have the identity $(n=1$ here $)$

$$
\begin{aligned}
\nabla^{*}(f & \left.D+\sum_{s=1}^{3} d h\left(\xi_{s}\right) F_{s}+4 \sum_{s=1}^{3} d h\left(\xi_{s}\right) I_{s} A_{s}-\frac{10}{3} \sum_{s=1}^{3} d h\left(\xi_{s}\right) I_{s} A\right) \\
= & \left(d h\left(e_{a}\right)-\frac{1}{4} h^{-2} d h\left(e_{a}\right)|\nabla h|^{2}+\frac{1}{2} h^{-1} \nabla d h\left(e_{a}, \nabla h\right)\right) D\left(e_{a}\right) \\
& +f\left(\left|T^{0}\right|^{2}-h^{-1} d h\left(e_{a}\right) D\left(e_{a}\right)-h^{-1}(n+2) d h\left(e_{a}\right) A\left(e_{a}\right)\right) \\
& +\sum_{s=1}^{3} \nabla d h\left(I_{s} e_{a}, \xi_{s}\right) F_{s}\left(I_{s} e_{a}\right) \\
& +h^{-1} \sum_{s=1}^{3}\left[d h\left(\xi_{s}\right) d h\left(I_{s} e_{a}\right) D\left(e_{a}\right)+(n+2) d h\left(\xi_{s}\right) d h\left(I_{s} e_{a}\right) A\left(e_{a}\right)\right] \\
& +4 \sum_{s=1}^{3} \nabla d h\left(I_{s} e_{a}, \xi_{s}\right) A_{s}\left(e_{a}\right)-\frac{10}{3} \sum_{s=1}^{3} \nabla d h\left(I_{s} e_{a}, \xi_{s}\right) A\left(e_{a}\right) \\
= & \left(d h\left(e_{a}\right)-\frac{1}{4} h^{-2} d h\left(e_{a}\right)|\nabla h|^{2}+\frac{1}{2} h^{-1} \nabla d h\left(e_{a}, \nabla h\right)\right) \sum_{t=1}^{3} D_{t}\left(e_{a}\right) \\
& +f\left(\left|T^{0}\right|^{2}-h^{-1} d h\left(e_{a}\right)\right)\left(\sum_{t=1}^{3} D_{t}\left(e_{a}\right)\right)-f h^{-1}(n+2) d h\left(e_{a}\right)\left(\sum_{t=1}^{3} A_{t}\left(e_{a}\right)\right) \\
& +\nabla d h\left(I_{1} e_{a}, \xi_{1}\right)\left(D_{1}\left(e_{a}\right)-D_{2}\left(e_{a}\right)-D_{3}\left(e_{a}\right)\right) \\
& +\nabla d h\left(I_{2} e_{a}, \xi_{2}\right)\left(-D_{1}\left(e_{a}\right)+D_{2}\left(e_{a}\right)-D_{3}\left(e_{a}\right)\right) \\
& +\nabla d h\left(I_{3} e_{a}, \xi_{3}\right)\left(-D_{1}\left(e_{a}\right)-D_{2}\left(e_{a}\right)+D_{3}\left(e_{a}\right)\right) \\
& +h^{-1}\left(\sum_{s=1}^{3} d h\left(\xi_{s}\right) d h\left(I_{s} e_{a}\right)\right)\left(\sum_{t=1}^{3} D_{t}\left(e_{a}\right)\right) \\
& +h^{-1}(n+2)\left(\sum_{s=1}^{3} d h\left(\xi_{s}\right) d h\left(I_{s} e_{a}\right)\right)\left(\sum_{t=1}^{3} A_{t}\left(e_{a}\right)\right) \\
& +4 \sum_{s=1}^{3} \nabla d h\left(I_{s} e_{a}, \xi_{s}\right) A_{s}\left(e_{a}\right)-\frac{10}{3}\left(\sum_{s=1}^{3} \nabla d h\left(I_{s} e_{a}, \xi_{s}\right)\right)\left(\sum_{t=1}^{3} A_{t}\left(e_{a}\right)\right) \\
&
\end{aligned}
$$


where the last equality uses 4.6 to express the vectors $F_{s}$ by $D_{s}$, and the expansions of the vectors $A$ and $D$ according to (3.5) and (4.5). Since the dimension of $M$ is seven it follows that $U=\bar{U}=\left[\nabla d h-2 h^{-1} d h \otimes d h\right]_{[3][0]}=0$. This, together with the Yamabe equation (3.4), which when $n=1$ becomes $\Delta h=2-4 h+3 h^{-1}|\nabla h|^{2}$, yields the formula (cf. 3.13)

$$
\nabla d h(X, \nabla h)+\sum_{s=1}^{3} \nabla d h\left(I_{s} X, I_{s} \nabla h\right)-\left(2-4 h+3 h^{-1}|\nabla h|^{2}\right) d h(X)=0 .
$$

From equations (4.4) and 3.12) we have

$$
\begin{aligned}
& D_{1}(X)=h^{-2} d h\left(\xi_{1}\right) d h\left(I_{1} X\right)+\frac{1}{4} h^{-2}\left[\nabla d h(X, \nabla h)+\nabla d h\left(I_{1} X, I_{1} \nabla h\right)\right. \\
& \left.-\nabla d h\left(I_{2} X, I_{2} \nabla h\right)-\nabla d h\left(I_{3} X, I_{3} \nabla h\right)\right], \\
& D_{2}(X)=h^{-2} d h\left(\xi_{2}\right) d h\left(I_{2} X\right)+\frac{1}{4} h^{-2}\left[\nabla d h(X, \nabla h)-\nabla d h\left(I_{1} X, I_{1} \nabla h\right)\right. \\
& \left.+\nabla d h\left(I_{2} X, I_{2} \nabla h\right)-\nabla d h\left(I_{3} X, I_{3} \nabla h\right)\right], \\
& D_{3}(X)=h^{-2} d h\left(\xi_{3}\right) d h\left(I_{3} X\right)+\frac{1}{4} h^{-2}\left[\nabla d h(X, \nabla h)-\nabla d h\left(I_{1} X, I_{1} \nabla h\right)\right. \\
& \left.-\nabla d h\left(I_{2} X, I_{2} \nabla h\right)+\nabla d h\left(I_{3} X, I_{3} \nabla h\right)\right] .
\end{aligned}
$$

Expressing the first term in 4.9 by the rest and substituting the result in the above equations we come to

$$
\begin{aligned}
D_{i}\left(e_{a}\right)= & \frac{1}{4} h^{-2}\left(2-4 h+3 h^{-1}|\nabla h|^{2}\right) d h\left(e_{a}\right)+h^{-2} d h\left(\xi_{i}\right) d h\left(I_{i} e_{a}\right) \\
& +\frac{1}{2} h^{-2}\left[-\nabla d h\left(I_{j} e_{a}, I_{j} \nabla h\right)-\nabla d h\left(I_{k} e_{a}, I_{k} \nabla h\right)\right] .
\end{aligned}
$$

At this point, by a purely algebraic calculation, using Lemma 3.1 and 4.10 we find

$$
\begin{aligned}
\frac{22}{3} A_{1}- & \frac{2}{3} A_{2}-\frac{2}{3} A_{3}+\frac{11}{3} D_{1}-\frac{1}{3} D_{2}-\frac{1}{3} D_{3} \\
= & -3 h^{-1}\left(1+\frac{1}{2} h^{-1} d h\left(e_{a}\right)+\frac{1}{4} h^{-2}|\nabla h|^{2}\right) d h\left(e_{a}\right)+3 h^{-2}\left(\sum_{s=1}^{3} d h\left(\xi_{s}\right) d h\left(I_{s} e_{a}\right)\right) \\
& +\frac{2}{3} h^{-1} \nabla d h\left(I_{1} e_{a}, \xi_{1}\right)-\frac{10}{3} h^{-1} \nabla d h\left(I_{2} e_{a}, \xi_{2}\right)-\frac{10}{3} h^{-1} \nabla d h\left(I_{3} e_{a}, \xi_{3}\right) .
\end{aligned}
$$

Similarly,

$$
\begin{aligned}
3 A_{1}-A_{2}- & A_{3}+2 D_{1} \\
= & \left(-2 h^{-1}+\frac{1}{2} h^{-2}+h^{-3}|\nabla h|^{2}\right) d h\left(e_{a}\right)-\frac{1}{2} h^{-2} \sum_{s=1}^{3} \nabla d h\left(I_{s} e_{a}, I_{s} \nabla h\right) \\
& +h^{-1} \nabla d h\left(I_{1} e_{a}, \xi_{1}\right)-h^{-1} \nabla d h\left(I_{2} e_{a}, \xi_{2}\right) \\
& -h^{-1} \nabla d h\left(I_{3} e_{a}, \xi_{3}\right)+h^{-2} \sum_{s=1}^{3} d h\left(\xi_{s}\right) d h\left(I_{s} e_{a}\right) .
\end{aligned}
$$


On the other hand, the coefficient of $A_{1}\left(e_{a}\right)$ in 4.8 is found to be, after setting $n=1$,

$$
\begin{aligned}
h\left[-3\left(1+\frac{1}{2} h^{-1}\right.\right. & \left.+\frac{1}{4} h^{-2}|\nabla h|^{2}\right) h^{-1} d h\left(e_{a}\right)+3 h^{-2}\left(\sum_{s=1}^{3} d h\left(\xi_{s}\right) d h\left(I_{s} e_{a}\right)\right) \\
& \left.+\frac{2}{3} h^{-1} \nabla d h\left(I_{1} e_{a}, \xi_{1}\right)-\frac{10}{3} h^{-1} \nabla d h\left(I_{2} e_{a}, \xi_{2}\right)-\frac{10}{3} h^{-1} \nabla d h\left(I_{3} e_{a}, \xi_{3}\right)\right],
\end{aligned}
$$

while the coefficient of $D_{1}\left(e_{a}\right)$ in 4.8$)$ is

$$
\begin{aligned}
d h\left(e_{a}\right)-\frac{1}{4} h^{-2} d h\left(e_{a}\right)|\nabla h|^{2}+\frac{1}{2} h^{-1} \nabla d h\left(e_{a}, \nabla h\right)-f h^{-1} d h\left(e_{a}\right) \\
+\nabla d h\left(I_{1} e_{a}, \xi_{1}\right)-\nabla d h\left(I_{2} e_{a}, \xi_{2}\right)-\nabla d h\left(I_{3} e_{a}, \xi_{3}\right) D_{1}\left(e_{a}\right) \\
+h^{-1}\left(\sum_{s=1}^{3} d h\left(\xi_{s}\right) d h\left(I_{s} e_{a}\right)\right) .
\end{aligned}
$$

Substituting $\nabla d h\left(e_{a}, \nabla h\right)=-\sum_{s=1}^{3} \nabla d h\left(I_{s} e_{a}, I_{s} \nabla h\right)+\left(2-4 h+3 h^{-1}|\nabla h|^{2}\right) d h\left(e_{a}\right)$ according to 4.9 and using the definition of $f$ transforms the above expression into

$$
\begin{aligned}
d h\left(e_{a}\right)-\frac{1}{4} h^{-2} d h\left(e_{a}\right)|\nabla h|^{2}-\left(\frac{1}{2}+h+\frac{1}{4} h^{-1}|\nabla h|^{2}\right) h^{-1} d h\left(e_{a}\right) \\
+\frac{1}{2} h^{-1}\left(-\sum_{s=1}^{3} \nabla d h\left(I_{s} e_{a}, I_{s} \nabla h\right)+\left(2-4 h+3 h^{-1}|\nabla h|^{2}\right) d h\left(e_{a}\right)\right) \\
+\nabla d h\left(I_{1} e_{a}, \xi_{1}\right)-\nabla d h\left(I_{2} e_{a}, \xi_{2}\right)-\nabla d h\left(I_{3} e_{a}, \xi_{3}\right) D_{1}\left(e_{a}\right) \\
+h^{-1}\left(\sum_{s=1}^{3} d h\left(\xi_{s}\right) d h\left(I_{s} e_{a}\right)\right) .
\end{aligned}
$$

Simplifying the above expression shows that the coefficient of $D_{1}\left(e_{a}\right)$ in 4.8 is

$$
\begin{aligned}
(-2+ & \left.\frac{1}{2} h^{-1}+h^{-2}|\nabla h|^{2}\right) d h\left(e_{a}\right)-\frac{1}{2} h^{-1}\left(\sum_{s=1}^{3} \nabla d h\left(I_{s} e_{a}, I_{s} \nabla h\right)\right) \\
& +\nabla d h\left(I_{1} e_{a}, \xi_{1}\right)-\nabla d h\left(I_{2} e_{a}, \xi_{2}\right)-\nabla d h\left(I_{3} e_{a}, \xi_{3}\right)+h^{-1}\left(\sum_{s=1}^{3} d h\left(\xi_{s}\right) d h\left(I_{s} e_{a}\right)\right) .
\end{aligned}
$$

Hence, we proved that the coefficient of $D_{1}\left(e_{a}\right)$ in 4.8$)$ is $h\left(3 A_{1}-A_{2}-A_{3}+\right.$ $\left.2 D_{1}\right)\left(e_{a}\right)$, while that of $A_{1}\left(e_{a}\right)$ is $h\left(\frac{22}{3} A_{1}-\frac{2}{3} A_{2}-\frac{2}{3} A_{3}+\frac{1}{3} D_{1}-\frac{1}{3} D_{2}-\frac{1}{3} D_{3}\right)\left(e_{a}\right)$. A cyclic permutation gives the rest of the coefficients in (4.8). With this, the divergence 4.8) can be written in the form

$$
\begin{aligned}
& \nabla^{*}\left(f D+\sum_{s=1}^{3} d h\left(\xi_{s}\right) F_{s}+4 \sum_{s=1}^{3} d h\left(\xi_{s}\right) I_{s} A_{s}-\frac{10}{3} \sum_{s=1}^{3} d h\left(\xi_{s}\right) I_{s} A\right) \\
&=f\left|T^{0}\right|^{2}+ h \sigma_{1,2,3}\left\{g\left(D_{1}, 3 A_{1}-A_{2}-A_{3}+2 D_{1}\right)\right. \\
&\left.+g\left(A_{1}, \frac{22}{3} A_{1}-\frac{2}{3} A_{2}-\frac{2}{3} A_{3}+\frac{11}{3} D_{1}-\frac{1}{3} D_{2}-\frac{1}{3} D_{3}\right)\right\}
\end{aligned}
$$


where $\sigma_{1,2,3}$ denotes the sum over all positive permutations of $(1,2,3)$. Let $Q$ be equal to

$$
Q:=\left[\begin{array}{cccccc}
2 & 0 & 0 & \frac{10}{3} & -\frac{2}{3} & -\frac{2}{3} \\
0 & 2 & 0 & -\frac{2}{3} & \frac{10}{3} & -\frac{2}{3} \\
0 & 0 & 2 & -\frac{2}{3} & -\frac{2}{3} & \frac{10}{3} \\
\frac{10}{3} & -\frac{2}{3} & -\frac{2}{3} & \frac{22}{3} & -\frac{2}{3} & -\frac{2}{3} \\
-\frac{2}{3} & \frac{10}{3} & -\frac{2}{3} & -\frac{2}{3} & \frac{22}{3} & -\frac{2}{3} \\
-\frac{2}{3} & -\frac{2}{3} & \frac{10}{3} & -\frac{2}{3} & -\frac{2}{3} & \frac{22}{3}
\end{array}\right]
$$

so that

$$
\nabla^{*}\left(f D+\sum_{s=1}^{3} d h\left(\xi_{s}\right) F_{s}+4 d h\left(\xi_{s}\right) I_{s} A_{s}-\frac{10}{3} \sum_{s=1}^{3} d h\left(\xi_{s}\right) I_{s} A\right)=f\left|T^{0}\right|^{2}+h\langle Q V, V\rangle
$$

with $V=\left(D_{1}, D_{2}, D_{3}, A_{1}, A_{2}, A_{3}\right)$. It is not hard to see that the eigenvalues of $Q$ are given by

$$
\{0,0,2(2+\sqrt{2}), 2(2-\sqrt{2}), 10,10\},
$$

which shows that $Q$ is a non-negative matrix.

\section{Proofs of the main theorems}

The proofs rely on Theorem 4.4 and the following characterization of all qc-Einstein structures conformal to the standard qc structures on the Heisenberg group.

Theorem 5.1 ([IMV], Theorem 1.2]). Let $\Theta=\frac{1}{2 h} \tilde{\Theta}$ be a conformal deformation of the standard qc-structure $\tilde{\Theta}$ on the quaternionic Heisenberg group $\boldsymbol{G}(\mathbb{H})$. Then $\Theta$ is qcEinstein if and only if, up to a left translation, the function $h$ is given by

$$
h=c\left[\left(1+v|q|^{2}\right)^{2}+v^{2}\left(x^{2}+y^{2}+z^{2}\right)\right],
$$

where $c$ and $v$ are any positive constants.

Consider first the case of the (seven-dimensional) sphere.

\subsection{Proof of Theorem 1.1}

Integrating the divergence formula of Theorem 4.4 we see that according to the divergence theorem established in [IMV, Proposition 8.1] the integral of the left-hand side is zero. Thus, the right-hand side vanishes as well, which shows that the quaternionic contact structure $\eta$ has vanishing torsion, i.e., it is also qc-Einstein according to Theorem 2.4

Next we bring into consideration the 7-dimensional quaternionic Heisenberg group and the quaternionic Cayley transform as described in [IMV, Section 5.2]. The quaternionic Heisenberg group of dimension 7 is $\boldsymbol{G}(\mathbb{H})=\mathbb{H} \times \operatorname{Im} \mathbb{H}$. The group law is given 
by $\left(q^{\prime}, \omega^{\prime}\right)=\left(q_{0}, \omega_{0}\right) \circ(q, \omega)=\left(q_{0}+q, \omega+\omega_{0}+2 \operatorname{Im} q_{0} \bar{q}\right)$, where $q, q_{0} \in \mathbb{H}$ and $\omega, \omega_{0} \in \operatorname{Im} \mathbb{H}$. The left-invariant orthonormal basis of the horizontal space is

$$
\begin{array}{ll}
T_{1}=\frac{\partial}{\partial t_{1}}+2 x^{1} \frac{\partial}{\partial x}+2 y^{1} \frac{\partial}{\partial y}+2 z^{1} \frac{\partial}{\partial z}, & X_{1}=\frac{\partial}{\partial x_{1}}-2 t^{1} \frac{\partial}{\partial x}-2 z^{1} \frac{\partial}{\partial y}+2 y^{1} \frac{\partial}{\partial z}, \\
Y_{1}=\frac{\partial}{\partial y_{1}}+2 z^{1} \frac{\partial}{\partial x}-2 t^{1} \frac{\partial}{\partial y}-2 x^{1} \frac{\partial}{\partial z}, & Z_{1}=\frac{\partial}{\partial z_{1}}-2 y^{1} \frac{\partial}{\partial x}+2 x^{1} \frac{\partial}{\partial y}-2 t^{1} \frac{\partial}{\partial z},
\end{array}
$$

using $q=t_{1}+i x_{1}+j y_{1}+k z_{1}$ and $\omega=i x+j y+k z$. The central (vertical) orthonormal vector fields $\xi_{1}, \xi_{2}, \xi_{3}$ are described as follows:

$$
\xi_{1}=2 \frac{\partial}{\partial x}, \quad \xi_{2}=2 \frac{\partial}{\partial y}, \quad \xi_{3}=2 \frac{\partial}{\partial z} .
$$

Let us identify the (seven-dimensional) group $G(\mathbb{H})$ with the boundary $\Sigma$ of a Siegel domain in $\mathbb{H} \times \mathbb{H}$,

$$
\Sigma=\left\{\left(q^{\prime}, p^{\prime}\right) \in \mathbb{H} \times \mathbb{H}: \Re p^{\prime}=\left|q^{\prime}\right|^{2}\right\} .
$$

$\Sigma$ carries a natural group structure and the map $(q, \omega) \mapsto\left(q,|q|^{2}-\omega\right) \in \Sigma$ is an isomorphism between $\boldsymbol{G}(\mathbb{H})$ and $\Sigma$.

The standard contact form, written as a purely imaginary quaternion valued form, on $\boldsymbol{G}(\mathbb{H})$ is given by $2 \tilde{\Theta}=(d \omega-q \cdot d \bar{q}+d q \cdot \bar{q})$, where $\cdot$ denotes quaternion multiplication. Since $d p=q \cdot d \bar{q}+d q \cdot \bar{q}-d \omega$, under the identification of $\boldsymbol{G}(\mathbb{H})$ with $\Sigma$ we also have $2 \tilde{\Theta}=-d p^{\prime}+2 d q^{\prime} \cdot \bar{q}^{\prime}$. Taking into account that $\tilde{\Theta}$ is purely imaginary, the last equation can also be written in the following form:

$$
4 \tilde{\Theta}=\left(d \bar{p}^{\prime}-d p^{\prime}\right)+2 d q^{\prime} \cdot \bar{q}^{\prime}-2 q^{\prime} \cdot d \bar{q}^{\prime} .
$$

The (quaternionic) Cayley transform is the map $\mathcal{C}: S \backslash\{(-1,0)\} \rightarrow \Sigma$ from the sphere $S=\left\{(q, p) \in \mathbb{H} \times \mathbb{H}:|q|^{2}+|p|^{2}=1\right\} \subset \mathbb{H} \times \mathbb{H}$ minus a point to the Heisenberg group $\Sigma=\left\{\left(q_{1}, p_{1}\right) \in \mathbb{H} \times \mathbb{H}: \Re p_{1}=\left|q_{1}\right|^{2}\right\}$, with $\mathcal{C}$ defined by

$$
\left(q_{1}, p_{1}\right)=\mathcal{C}((q, p)), \quad q_{1}=(1+p)^{-1} q, \quad p_{1}=(1+p)^{-1}(1-p)
$$

with inverse $(q, p)=\mathcal{C}^{-1}\left(\left(q_{1}, p_{1}\right)\right)$ given by

$$
q=2\left(1+p_{1}\right)^{-1} q_{1}, \quad p=\left(1-p_{1}\right)\left(1+p_{1}\right)^{-1} .
$$

The Cayley transform is a conformal quaternionic contact diffeomorphism between the quaternionic Heisenberg group with its standard quaternionic contact structure $\tilde{\Theta}$ and $S \backslash\{(-1,0)\}$ with its standard structure $\tilde{\eta}$ (see [IMV]),

$$
\lambda \cdot\left(\mathfrak{C}_{*} \tilde{\eta}\right) \cdot \bar{\lambda}=\frac{8}{\left|1+p_{1}\right|^{2}} \tilde{\Theta},
$$

where $\lambda=\left(1+p_{1}\right) /\left(\left|1+p_{1}\right|\right)$ is a unit quaternion and $\tilde{\eta}$ is the standard quaternionic contact form on the sphere, $\tilde{\eta}=d q \cdot \bar{q}+d p \cdot \bar{p}-q \cdot d \bar{q}-p \cdot d \bar{p}$. Hence, up to a constant multiplicative factor and a quaternionic contact automorphism the forms $\mathcal{C}_{*} \tilde{\eta}$ and $\tilde{\Theta}$ are conformal to each other. It follows that the same is true for $\mathcal{E}_{*} \eta$ and $\tilde{\Theta}$. In 
addition, $\tilde{\Theta}$ is qc-Einstein by definition, while $\eta$ and hence also $\mathcal{C}_{*} \eta$ are qc-Einstein as we observed at the beginning of the proof. According to Theorem 5.1, up to a multiplicative constant factor, the forms $\mathcal{C}_{*} \tilde{\eta}$ and $\mathcal{C}_{*} \eta$ are related by a translation or dilation on the Heisenberg group. Hence, we conclude that up to a multiplicative constant, $\eta$ is obtained from $\tilde{\eta}$ by a conformal quaternionic contact automorphism, which proves the first claim of Theorem 1.1. From the conformal properties of the Cayley transform and [Va2, Va1] it follows that the minimum $\lambda\left(S^{4 n+3}\right)$ is achieved by a smooth 3-contact form, which due to the Yamabe equation is of constant qc-scalar curvature. This shows the second claim of Theorem 1.1 .

\subsection{Proof of Theorem 1.3}

Let $\mathcal{D}^{1,2}$ be the space of functions $u \in L^{2^{*}}(\boldsymbol{G}(\mathbb{H}))$ having distributional horizontal gradient $|\nabla u|^{2}=\left|T_{1} u\right|^{2}+\left|X_{1} u\right|^{2}+\left|Y_{1} u\right|^{2}+\left|Z_{1} u\right|^{2} \in L^{2}(G(\mathbb{H}))$ with respect to the Lebesgue measure $d H$ on $\mathbb{R}^{7}$, which is the Haar measure on the group. Let us define the constant $\left(2^{*}=5 / 2\right.$ here $)$

$$
\Lambda:=\inf \left\{\int_{\boldsymbol{G}(\mathbb{H})}|\nabla v|^{2} d H: v \in \mathcal{D}^{1,2}, v \geq 0, \int_{\boldsymbol{G}(\mathbb{H})}|v|^{2^{*}} d H=1\right\} .
$$

Let $v$ be a function for which the infimum is achieved. Note that such a function exists by [Va2] or [Va1]. Furthermore, $\Lambda=S_{2}^{-2}$, where $S_{2}$ is the best constant in the $L^{2}$ FollandStein inequality (1.1), since $v \in \mathcal{D}^{1,2}$ implies $|v| \in \mathcal{D}^{1,2}$ and the gradient is the same a.e. From the choice of $v$ we have

$$
\Lambda=\int_{\boldsymbol{G}(\mathbb{H})}|\nabla v|^{2} d H, \quad \int_{\boldsymbol{G}(\mathbb{H})} v^{2^{*}} d H=1 .
$$

Writing the Euler-Lagrange equation of the constrained problem we see that $v$ is a nonnegative entire solution of $\left(T_{1}^{2}+X_{1}^{2}+Y_{1}^{2}+Z_{1}^{2}\right) v=-\Lambda v^{3 / 2}$. By [GV2, Lemma 10.2] ( see [Va1] or [Va2, Theorem 10.3] for further details), $v$ is a bounded function. Similarly to [FSt, Theorem 16.7] it follows $v$ is a Lipschitz continuous function in the sense of non-isotropic Lipschitz spaces $[\mathrm{F}]$. Iterating this argument and using $[\mathrm{F}$, Theorem 5.25] we see that $v$ is a $C^{\infty}$ smooth function on the set where it is positive, while being of class $\Gamma_{\text {loc }}^{2, \beta}$, the non-isotropic Lipschitz space, for some $\beta>0$. In particular $v$ is continuously differentiable function by [F, Theorem 5.25]. Applying the Hopf lemma [GV1, Theorem 2.13] on the set where $v$ is positive shows that $v$ cannot vanish, i.e., it is a positive entire solution to the Yamabe equation. The positivity can also be seen by the Harnack inequality (see [W] for example). Let $u=\Lambda^{1 /\left(2^{*-2}\right)} v$. Then $u$ is a positive entire solution of the Yamabe equation

$$
\left(T_{1}^{2}+X_{1}^{2}+Y_{1}^{2}+Z_{1}^{2}\right) u=-u^{3 / 2} .
$$

From the definition of $u$, we have

$$
\Lambda=\left(\int_{\boldsymbol{G}(\mathbb{H})}|\nabla u|^{2} d H\right)^{1 / 5}=\left(\int_{\boldsymbol{G}(\mathbb{H})} u^{5 / 2} d H\right)^{1 / 5} .
$$


We shall compute the last integral by determining $u$ with the help of the divergence formula.

As before, let $\tilde{\Theta}$ be the standard contact form on $G(\mathbb{H})$ identified with $\Sigma$. Using the inversion and the Kelvin transform on $\boldsymbol{G}(\mathbb{H})$ (cf. [GV2, Sections 8 and 9]), we can see that if $\Theta=\frac{1}{2 h} \tilde{\Theta}$ has constant scalar curvature, then the Cayley transform lifts the qc structure defined by $\Theta$ to a qc structure of constant qc-scalar curvature on the sphere, which is conformal to the standard one. The details are as follows. Let us define two contact forms $\Theta_{1}$ and $\Theta_{2}$ on $\Sigma$ setting

$$
\Theta_{1}=u^{4 /(Q-2)} \tilde{\Theta} \quad \text { and } \quad \Theta_{2}=(\mathcal{K} u)^{4 /(Q-2)} \frac{\bar{p}^{\prime}}{\left|p^{\prime}\right|} \tilde{\Theta} \frac{p^{\prime}}{\left|p^{\prime}\right|},
$$

where $u$ is as in (5.5), $\mathcal{K} u$ is its Kelvin transform (see (5.8) below for the exact formula), and $Q$ is the homogeneous dimension of the group. Notice that $\frac{\bar{p}^{\prime}}{\left|p^{\prime}\right|} \tilde{\Theta} \frac{p^{\prime}}{\left|p^{\prime}\right|}$ defines the same qc structure on the Heisenberg group as $\tilde{\Theta}$, and $\mathcal{K} u$ is a smooth function on the whole group according to [GV2, Theorem 9.2]. We are going to see that using the Cayley transform these two contact forms define a contact form on the sphere, which is conformal to the standard one and has constant qc-scalar curvature.

Let $P_{1}=(-1,0)$ and $P_{2}=(1,0)$ be respectively the 'south' and 'north' poles of the unit sphere $S=\left\{|q|^{2}+|p|^{2}=1\right\}$. Let $\mathcal{C}_{1}$ and $\mathcal{C}_{2}$ be the corresponding Cayley transforms defined, respectively, on $S \backslash\left\{P_{1}\right\}$ and $S \backslash\left\{P_{2}\right\}$. Note that $\mathcal{C}_{1}$ was defined in [5.2), while $\mathrm{C}_{2}$ is given by

$$
\left(q_{2}, p_{2}\right)=\mathcal{C}_{2}((q, p)), \quad q_{2}=-(1-p)^{-1} q, \quad p_{2}=(1-p)^{-1}(1+p) .
$$

In order that $\Theta_{1}$ and $\Theta_{2}$ define a contact form $\eta$ on the sphere it is enough to see that

$$
\Theta_{1}(p, q)=\Theta_{2} \circ \mathcal{C}_{2} \circ \mathcal{C}_{1}^{-1}(p, q), \quad \text { i.e., } \quad \Theta_{1}=\left(\mathcal{C}_{2} \circ \mathcal{C}_{1}^{-1}\right)^{*} \Theta_{2} .
$$

A calculation shows that $\mathcal{C}_{2} \circ \mathcal{C}_{1}^{-1}: \Sigma \rightarrow \Sigma$ is given by

$$
q_{2}=-p_{1}^{-1} q_{1}, \quad p_{2}=p_{1}^{-1},
$$

or equivalently, in the model $G(\mathbb{H})$,

$$
q_{2}=-\left(\left|q_{1}\right|^{2}-\omega_{1}\right)^{-1} q_{1}, \quad \omega_{2}=-\frac{\omega_{1}}{\left|q_{1}\right|^{4}+\left|\omega_{1}\right|^{2}} .
$$

Hence, $\sigma=\mathcal{C}_{2} \circ \mathcal{C}_{1}^{-1}$ is an involution on the group. Furthermore, with the help of 5.4 we calculate

$$
\mathfrak{C}_{1 *} \circ \mathcal{C}_{2} * \Theta=\frac{1}{\left|p_{1}\right|^{2}} \bar{\mu} \Theta \mu, \quad \mu=\frac{p_{1}}{\left|p_{1}\right|},
$$

which proves the identity [5.7]. Using the properties of the Kelvin transform [GV2, Sections 8 and 9$]$

$$
(\mathcal{K} u)\left(q^{\prime}, p^{\prime}\right):=\left|p^{\prime}\right|^{-(Q-2) / 2} u\left(\sigma\left(q^{\prime}, p^{\prime}\right)\right),
$$


we see that $u$ and $\mathcal{K} u$ are solutions of the Yamabe equation 5.5. This implies that the contact form $\eta$ has constant qc-scalar curvature, equal to $4(Q+2) /(Q-2)$.

Notice that $\eta$ is conformal to the standard form $\tilde{\eta}$ and the arguments in the preceding proof imply that $\eta$ is qc-Einstein. A small calculation shows that this is equivalent to the fact that if we set

$$
\bar{u}=2^{10}\left[\left(1+|q|^{2}\right)^{2}+|\omega|^{2}\right]^{-2},
$$

then $\bar{u}$ satisfies the Yamabe equation (5.5) and all other non-negative solutions of (5.5) in the space $\mathcal{D}^{1,2}$ are obtained from $\bar{u}$ by translations and dilations,

$$
\begin{gathered}
\tau_{\left(q_{0}, \omega_{0}\right)} \bar{u}(q, \omega):=\bar{u}\left(q_{0}+q, \omega+\omega_{0}\right), \\
\bar{u}_{\lambda}(q):=\lambda^{4} \bar{u}\left(\lambda q, \lambda^{2} \omega\right), \quad \lambda>0 .
\end{gathered}
$$

Thus $u$, which was defined at the beginning of the proof, is given by equation (5.9) up to translations and dilations. This allows the calculation of the best constant in the FollandStein inequality (see [GV1, (4.52)])

$$
\Lambda^{5}=\int_{G(\mathbb{H})} \frac{2^{25}}{\left.\left[\left(1+|q|^{2}\right)^{2}+|\omega|^{2}\right)\right]^{5}} d H=2^{25} \pi^{7 / 2} \frac{\Gamma(7 / 2)}{\Gamma(7)}=\frac{\pi^{12 / 10}}{12},
$$

where $\Gamma$ is the Gamma function. Hence

$$
S_{2}=\Lambda^{-1 / 2}=\frac{2 \sqrt{3}}{\pi^{3 / 5}} .
$$

Recalling the relation between $u$ and $v$ we find that the extremals in the Folland-Stein embedding are given by

$$
v=\frac{2^{11} \sqrt{3}}{\pi^{3 / 5}}\left[\left(1+|q|^{2}\right)^{2}+|\omega|^{2}\right]^{-2}
$$

and its translations and dilations. The proof of Theorem 1.3 is complete.

Acknowledgments. S. Ivanov was visiting Max-Planck-Institut für Mathematics, Bonn. S.I. thanks MPIM, Bonn for providing the support and an excellent research environment during the final stages of the paper. S.I. is a Senior Associate to the Abdus Salam ICTP. I. Minchev is a member of the Junior Research Group "Special Geometries in Mathematical Physics" founded by the Volkswagen Foundation. The authors would like to thank The National Academies for the financial support and University of California at Riverside and University of Sofia for hosting the respective visits of the authors.

The authors would like to thank the referee for remarks making the exposition clearer and spotting several typos in the paper.

This project has been funded in part by the National Academy of Sciences under the Collaboration in Basic Science and Engineering Program 1 Twinning Program supported by Contract No. INT-0002341 from the National Science Foundation. The contents of this publication do not necessarily reflect the views or policies of the National Academy of Sciences or the National Science Foundation, nor does mention of trade names, commercial products or organizations imply endorsement by the National Academy of Sciences or the National Science Foundation.

S.I. was partially supported by Contract 198/2010 with Sofia University. The three authors were partially supported by Contracts 'Idei', DO 02-257/2008 and DID 02-39/2009. 


\section{References}

[AK] Alekseevsky, D., Kamishima, Y.: Pseudo-conformal quaternionic CR structure on $(4 n+$ 3)-dimensional manifolds. Ann. Mat. Pura Appl. 187, 487-529 (2008) Zbl pre05292701 MR 2393145

[Biq1] Biquard, O.: Métriques d'Einstein asymptotiquement symétriques. Astérisque 265 (2000) Zbl 0967.53030 MR 1112625

[Biq2] Biquard, O.: Quaternionic contact structures. In: Quaternionic Structures in Mathematics and Physics (Rome, 1999), Univ. Roma "La Sapienza", Roma, 23-30 (1999) Zbl 0993.53017 MR 1848655

[CSal] Capria, M., Salamon, S.: Yang-Mills fields on quaternionic spaces. Nonlinearity 1, 517530 (1988) Zbl 0681.53037 MR 0967469

[D1] Duchemin, D.: Quaternionic contact structures in dimension 7. Ann. Inst. Fourier (Grenoble) 56, 851-885 (2006) Zbl 1122.53025 MR 2266881

[D2] Duchemin, D.: Quaternionic contact hypersurfaces. math.DG/0604147

[F] Folland, G.: Subelliptic estimates and function spaces on nilpotent Lie groups. Ark. Mat. 13, 161-207 (1975) Zbl 0312.35026 MR 0494315

[FSt] Folland, G. B., Stein, E. M.: Estimates for the $\bar{\partial}_{b}$ complex and analysis on the Heisenberg group. Comm. Pure Appl. Math. 27, 429-522 (1974) Zbl 0293.35012 MR 0367477

[GV1] Garofalo, N., Vassilev, D.: Symmetry properties of positive entire solutions of Yamabe type equations on groups of Heisenberg type. Duke Math. J. 106, 411-449 (2001) Zbl 1012.35014 MR 1813232

[GV2] Garofalo, N., Vassilev, D.: Regularity near the characteristic set in the non-linear Dirichlet problem and conformal geometry of sub-Laplacians on Carnot groups. Math. Ann. 318, 453-516 (2000) Zbl 1158.35341 MR 1800766

[IMV] Ivanov, St., Minchev, I., Vassilev, D.: Quaternionic contact Einstein structures and the quaternionic contact Yamabe problem. Preprint, math.DG/0611658

[IV] Ivanov, St., Vassilev, D.: Conformal quaternionic contact curvature and the local sphere theorem. J. Math. Pures Appl. 93, 277-307 (2010) Zbl pre05690746

[JL1] Jerison, D., Lee, J.: The Yamabe problem on CR manifolds. J. Differential Geom. 25, 167197 (1987) Zbl 0661.32026 MR 0880182

[JL2] Jerison, D., Lee, J.: Extremals for the Sobolev inequality on the Heisenberg group and the CR Yamabe problem. J. Amer. Math. Soc. 1, 1-13 (1988) Zbl 0634.32016 MR 0924699

[LP] Lee, J. M., Parker, T.: The Yamabe problem. Bull Amer. Math. Soc. 17, 37-91 (1987) Zbl 0633.53062 MR 0888880

[M] Mostow, G. D.: Strong Rigidity of Locally Symmetric Spaces. Ann. of Math. Stud. 78, Princeton Univ. Press, Princeton, NJ and Univ. of Tokyo Press, Tokyo (1973) Zbl 0265.53039 MR 0385004

[Ob] Obata, M.: The conjecture of conformal transformations in Riemannian manifolds. J. Differential Geom. 6, 247-258 (1971) Zbl 0236.53042 MR 0303464

[P] Pansu, P.: Métriques de Carnot-Carathéodory et quasiisométries des espaces symétriques de rang un. Ann. of Math. 129, 1-60 (1989) Zbl 0678.53042 MR 0979599

[Va1] Vassilev, D.: Yamabe type equations on Carnot groups. Ph.D. thesis, Purdue Univ. (2000)

[Va2] Vassilev, D.: Existence of solutions and regularity near the characteristic boundary for sub-Laplacian equations on Carnot groups. Pacific J. Math. 227, 361-397 (2006) Zbl pre05190383 MR 2263021

[W] Wang, W.: The Yamabe problem on quaternionic contact manifolds. Ann. Mat. Pura Appl. 186, 359-380 (2007) Zbl 1150.53007 MR 2295125 